\title{
TRACE SPECTRUM OF 1D TRANSFER MATRICES FOR WAVE PROPAGATION IN LAYERED MEDIA
}

\author{
J. GARCIA-SUAREZ
}

\begin{abstract}
The Transfer Matrix formalism is ubiquitous when it comes to study wave propagation in various stratified media, applications ranging from Seismology to Quantum Mechanics. A relation between variables at two points in two different layers can be established via a matrix, termed (global) transfer matrix (product of "atomic" single-layer matrices). As a matter of convenience, we focus on $1 \mathrm{D}$ propagation of axial waves in rods, but our results extend to other fields where the formalism applies. When the layering corresponds to a representative repeated cell in an otherwise infinitely periodic medium, the trace of the cumulative multi-layer matrix is known to control the dispersion relation. It is proved that this trace has a discrete spectrum made up of distinct $2^{N-1}$ harmonics (not necessarily orthogonal to each other in any sense) which are exactly characterized in terms of both the frequency of their oscillations and their amplitudes; the phase shift among harmonics is either zero or $\pi$. This definite appraisal of the discrete spectrum of the trace opens new strategies for rational design of bandgaps, going beyond parametric or sensitivity studies.
\end{abstract}

\section{INTRODUCTION}

1.1. Ubiquity of Transfer Matrix formalism. The Transfer Matrix method, in its two-variable variant, refers to an approach to solve two coupled scalar linear 1storder ODEs with two scalar unknowns, by, instead of looking to combine equations into one, recasting them into a single linear 1st-order vector ODE, where the entries of the unknown vector correspond to the original scalar unknowns.

Let us mention some of the branches of Physics and Engineering where this method has been applied, along with some useful references (the following partial list does not intend to be thorough, the reader is encouraged to explore the references included in the publications that are mentioned in the ensuing).

- Electromagnetic waves [1]: working with frequency-domain 1D Maxwell equations, one can relate (a) the amplitude and (b) the spatial derivative of the amplitude along the propagation direction, of either the electric or the magnetic field, at any positions in a stratified medium with different dielectric constant in each layer (such a structure is usually referred as "photonic crystal"). In particular, in Optics, the numerical approach to working with these matrices is termed "Abeles Method" and it is used to obtain global properties of the layering, as, for instance, its reflectivity.

- Heat transfer: the transfer matrix formalism also accommodates analysis of $1 \mathrm{D}$ heat fluxes in layered media [2]. This is an example of a parabolic

Date: November 2021. Affiliation: Civil Engineering Institute École Politechnique Fédérale de Lausanne (EPFL), Lausanne, Switzerland. 
problem (difussion) that fits the formalism, what highlights its application beyond the hyperbolic problems (wave propagation) that are the main theme of this article.

- Quantum Mechanics (Phononics): the time-independent 1D Schrodinger equation also admits a matrix representation [3], wherein the entries of the unknown vector are the wavefunction amplitude and its first derivative, the potential being discontinuous. The transfer matrix method induces a dynamical-system approach termed "trace maps" formalism [4]. Not only electronic properties affected by the stratification of the potential can be framed within this formalism, it also extends to lattice vibrations (phonons) and to quasi-periodic systems $[5,6]$.

- Acoustic waves: another field where it finds natural applications is the design of acoustic metamaterials [7]: consider a periodic medium where pressure waves propagate perpendicularly to the interface between layers; then, the product of the successive transfer matrices, each one of them defined for a different layer, relate the pressure value at any two locations along the longitudinal coordinate. Thus, this matrix encodes the periodicity of a system (indeed, the Bloch condition is expressed in terms of the entries of the multi-layer transfer matrix).

- Elastic waves: (a) in Seismology [8, 9], the methodology is employed to study a number of scenarios of wave traversing across strata, from simple 1D wave propagation (geotechnical engineers would talk of "1D Site Response Analysis" [10]) to inclined P-SV waves. In the context of both Seismology and Geotechnical Earthquake Engineering, these matrices tend to be referred as "propagators", "propagator matrices" or "matrizants" [11]. The goal of the analysis is, usually, to relate the fields at a certain depth to the ones at the ground surface, after the waves traverse across a number of layers of different soils.

(b) Laminates [12] are formally equivalent to the just mentioned soil strata. The main difference, despite formal similarities, is that one is also interested in understanding the effect of repeated layerings over the dispersion properties of the medium when assumed to be periodic. The study is tackled oftentimes in terms of either 1D compressional waves or shear waves. Cases other than propagation perpendicular to the layer edges have also been considered in the literature $[13,14]$.

(c) Axial wave propagation in rods with changing cross-section and/or material properties is yet another category that can be considered $[15,16]$. In general, also in this case, understanding the effect of periodic cells over the dispersion relation is the goal of the studies. See [17] for a recent review of applications of the method in Mechanical Engineering.

The latter configuration (longitudinal waves in heterogeneous rods) is the one we are to focus on in order to derive the new results, while some of our results may be generalizable to the other subjects which also employ the transfer matrix formalism in a different guise.

It should be obvious by now that this kind of problems has received, and still receives, the attention of many researches in different fields. Hence it should not come as a surprise that the exact expression of the general (any number of layers) 
entries of the propagator matrix have been partially stated [18, 7], yet its general, compact form is, to my knowledge, not available yet.

When it comes to design questions, parametric and sensitivity studies have been favored [19, 20], owing to the apparent complexity of the entries of the global transfer matrix for any number of layers greater than two.

1.2. Recent breakthrough: universal structure. In applications concerning elastic waves in laminates and rods, an elegant way of picturing the behavior of the dispersion relation by studying the winding on a toroidal surface has been proposed [12]. This approach is "universal" insofar it permits to represent the dispersion relation, for any kind of laminate or rod, over the same compact object: the surface of a torus over which a linear flow is defined. In essence, it consists of dividing the surface (or volume in higher dimensions) in two regions: one corresponding to passbands and another one to bandgaps. Ergodic theorems can then be used to relate averages over the frequency range to averages over the surface.

In [12], this representation was first staged using 2-component periodic laminates, where shear waves propagate. The extension of the theory from small strains to small-strain waves superimposed onto finite deformations was also introduced there for the first time.

A generalization to the case of $N$-layer laminates was attained in [18]. It was proved therein that higher-dimension tori were the proper means to adapt the results in [12] to more complex systems, and that many of the results carry on for any number of layers.

The framework was later adapted to the case of structured rods [16]. The toroidal representation was used to study a recurrent theme in this field: certain quasicrystalline structures called Fibonacci rods [15].

Remarkably, the aforementioned contributions did not require the knowledge of the exact form of the global transfer matrix for any number of layers, but just the interpretation of the arguments of the function controlling the dispersion relation as the flow over the toroidal surface.

Some relevant questions concerning the performance of periodic laminates were settled using this universal representation. These authors managed to:

- Identify what laminates yield periodic bandgaps.

- Compute the density of bandgaps in a given laminate over the whole frequency range (by computing the relative area of the no-propagation region).

- Explain to choose the relative width of the phases (e.g. choosing the slopes of the linear flow over the torus) in order to attain the widest first bandgap possible.

Closed-form expressions were obtained in some simple layerings, e.g. two-component cells, while the framework provided efficient approaches to answer these questions numerically in more complex ones.

The critical step that enabled obtaining these results is the delineation of the two regions the surface splits on (region corresponding to bandpass and the one to bandgap). One of the contributions of this manuscript is to outline the elementary blocks these regions are built from.

1.3. Structure of the text. Section 2 presents the physical system under scrutiny as well as the equations governing it. Section 3 introduces the main result: the general expression of the trace of the cumulative transfer matrix over the characteristic 


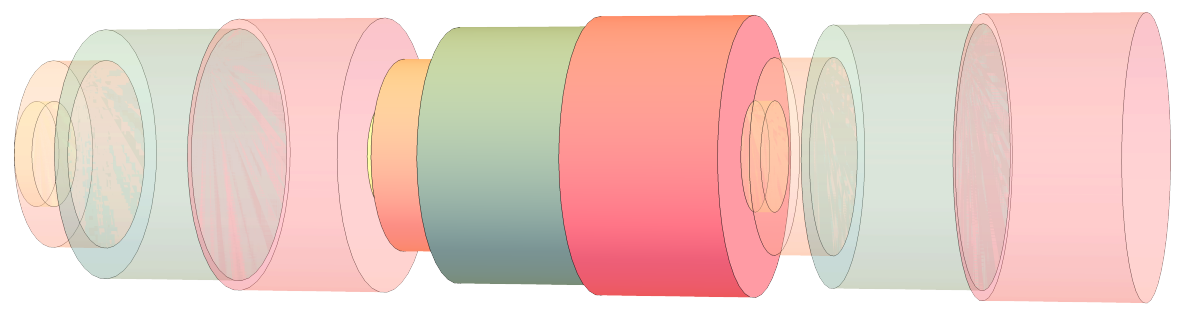

FiguRE 1. Scheme of the representative cell (highlighted) in a periodic rod to be subjected to axial wave propagation.

cell of a periodic rod. This expression encodes the "discrete spectrum of the dispersion relation" as a function of the geometric and material parameters. The function takes the wave frequency as independent variable and returns real values whose absolute value is less either than one (indicating a propagating wave for that frequency) or greater (evanescent wave, bandgap at that frequency). The section also includes verification of the result, while a detailed derivation has been consigned to the Appendix . Section 4 puts in display one logical manner of utilizing the newly-acquired knowledge of the spectrum to design a periodic rod whose first bandgap happens at the lowest possible frequency. Section 5 summarizes the main findings and lays out future research directions.

\section{Preliminaries}

2.1. The system. Figure 1 depicts the system: a representative cell made of $N$ layers of different material and cross-sections, with thicknesses $L_{i}, i=1, \ldots, N$, in an otherwise repetitive system assumed to be infinite.

The propagation of longitudinal waves in this system renders a $1 \mathrm{D}$ problem as the direction of propagation is perpendicular to all the interfaces between layers (and cross-section dispersive effects are not being considered [21]). The layer heterogeneity is described by the position-dependence in the cell of the Young's modulus, and

the cross-section area. Material properties and cross-section are assumed to remain constant in each layer. This system is similar to the ones studied in $[15,16]$.

2.2. Governing equations. In this scenario (rod axial waves, ignoring dispersive effects), the governing equations, in time domain, are

$$
\begin{aligned}
\frac{\partial}{\partial x}(\sigma A) & =\rho \frac{\partial^{2} u}{\partial t^{2}}, \\
\frac{\partial u}{\partial x} & =\frac{\sigma}{E},
\end{aligned}
$$

where $x$ is the longitudinal space coordinate, $t$ represents time, $\sigma$ is the normal stress to the interfaces, $u$ is the longitudinal displacement, $E$ is the Young modulus of the material the rod layer ("sub-rod") is made of, A is the cross-section area and $\rho$ must be understood as a linear density, i.e., mass per unit of length. Either by 
taking the Fourier transform or by assuming a plane-wave ansatz, the frequencydomain version of these equations are

$$
\begin{aligned}
\frac{d}{d x}(\hat{\sigma} A) & =-\rho \omega^{2} \hat{u}, \\
\frac{d \hat{u}}{d x} & =\frac{\hat{\sigma}}{E},
\end{aligned}
$$

where $\hat{u}$ and $\hat{\sigma}$ represent the amplitude of the displacement and the stress, respectively, and $\omega$ is the circular frequency. Recasting in matrix ODE form: for the $k$-th homogeneous portion [16],

$$
\frac{d \boldsymbol{f}}{d x}=\frac{d}{d x}\left[\begin{array}{c}
\hat{u} \\
\hat{\sigma} A
\end{array}\right]=\left[\begin{array}{cc}
0 & 1 / a_{k} \\
-\rho_{k} \omega^{2} & 0
\end{array}\right]\left[\begin{array}{c}
\hat{u} \\
\hat{\sigma} A
\end{array}\right]=\boldsymbol{A}_{k} \boldsymbol{f}(z),
$$

where $a_{k}=E_{k} A_{k}$ (do not confuse the $k$-th cross-section area, $A_{k}$ with the (boldface) $k$-th layer matrix $\left.\boldsymbol{A}_{k}\right)$. The solution, particularized at the end of the homogeneous sub-rod (whose length is $L_{k}$ ), is $\boldsymbol{f}\left(x_{k}\right)=\boldsymbol{T}_{k}\left(x_{k}, x_{k-1}\right) \boldsymbol{f}\left(x_{k-1}\right)$, where

$$
\boldsymbol{T}_{k}\left(x_{k}, x_{k-1}\right)=\exp \left(L_{k} \boldsymbol{A}_{k}\right)=\left[\begin{array}{cc}
\cos \left(\frac{\omega L_{k}}{c_{k}}\right) & \frac{\sin \left(\frac{\omega L_{k}}{c_{k}}\right)}{\sqrt{\rho_{k} a_{k}}} \\
-\sqrt{\rho_{k} a_{k}} \sin \left(\frac{\omega L_{k}}{c_{k}}\right) & \cos \left(\frac{\omega L_{k}}{c_{k}}\right)
\end{array}\right],
$$

and $c_{k}=\sqrt{a_{k} / \rho_{k}}$ is the wave velocity in the $k$-th layer.

By recursion, the motion-force vector $[\hat{u}, \hat{\sigma} A]^{\top}$ at any position in the cell (say, somewhere within the k-th layer) can be written in terms of the one at the initial point $\left(x=x_{0}\right)$, in particular to the end of the $N$-th layer, $x_{N}$ :

$$
\boldsymbol{f}\left(x_{N}\right)=\boldsymbol{T}\left(x_{N}, x_{0}\right) \boldsymbol{f}\left(x_{0}\right)=\boldsymbol{T}_{N}\left(x_{N}, x_{N-1}\right) \ldots T_{k}\left(x_{k}, x_{k-1}\right) \ldots T_{1}\left(x_{1}, x_{0}\right) \boldsymbol{f}\left(x_{0}\right) .
$$

2.3. Group-theoretical Aspects. It has already been noted elsewhere [5] that the transfer matrices represent elements of the special linear group $S L(2, \mathbb{R})$ [22].

The group $S L(2, \mathbb{R})$ is related to the Lorentz-like group $S O(2,1)[23]$, what allows to regard the propagator matrices as Lorentz-transformation (in 2D space), namely, the so-called "rapidity boosts".

This kind of group isomorphism belongs in the category termed "accidental" or "exceptional", as they arise as a consequence of the low-dimensionality of the ambient space being considered (two-by-two matrices), not from a genuine connection between groups that persists in higher dimensions.

These equivalences can be exploited nonetheless, the isomorphism to $S O(2,1)$ will be leveraged in order to derive exact expressions of the cumulative propagator, see the Appendix.

2.4. Periodicity and dispersion relations. Bloch condition [24] demands that $\boldsymbol{f}(x=L)=\boldsymbol{T}(L, 0) \boldsymbol{f}(x=0)=\exp (\mathrm{ik} L) \boldsymbol{f}(0)$ in the periodic cell, $\mathrm{k}$ being the wavenumber.

For the relation to have non-trivial solutions, $\exp (\mathrm{ik} L)$ must be an eigenvalue of $\boldsymbol{T}(L, 0)$. The equation for an eigenvalue, $\lambda$, is

$$
\lambda^{2}-\operatorname{trace}(\boldsymbol{T}(L, 0)) \lambda+1=0,
$$


where the fact that $\operatorname{det} \boldsymbol{T}(L, 0)=T_{22} T_{11}-T_{21} T_{12}=1$ (it is a matrix of $S L(2, \mathbb{R}$ ), after all) has been used. Substituting $\lambda=\exp (\mathrm{ik} L)$ in eq. (6), and then multiplying by $\exp (-\mathrm{ik} L)$, plus gathering terms, yields

$$
\cos (\mathrm{k} L)=\frac{1}{2} \operatorname{trace}(\boldsymbol{T}(L, 0)) .
$$

See that for as long as $|\operatorname{trace}(\boldsymbol{T}(L, 0))| \leq 2$, the prior transcendental equation admits real wavenumbers as solutions, so waves propagate at those frequencies, but if $|\operatorname{trace}(\boldsymbol{T}(L, 0))|>2$, the solution implies imaginary wavenumbers, thus the waves must decay in amplitude exponentially, no effective propagation takes place, and there would be a bandgap at that frequency.

2.5. A word on terminology. The word "spectrum" has been used $[12,18,16]$ to refer to the evolution of the function half-trace controlling the dispersion relation. In this text, we talk about a different concept: "the discrete spectrum" of this function, understood as the finite number of oscillating functions (modulated cosines, e.g., harmonics) it can be decomposed in.

\section{The TRACE EXACT FORMULA}

The half-trace function, $\eta(\omega)$ (this name is adopted following $[12,18]$ ), for a periodic cell made up of $N$ layers, admits the following form (see Appendix derivation leading to this result)

$$
\eta(\omega)=\frac{1}{2} \operatorname{trace}(\boldsymbol{T}(L, 0))=\sum_{k=1}^{2^{N-1}} \mathcal{T}_{k} \cos \left(\tau_{k} \omega\right),
$$

where the discrete spectrum of the half-trace of the laminate is fully defined by the characteristic "spectral periods" of the N-layer periodic cell, given by

$$
\tau_{j}=\left[\frac{L_{1}}{c_{1}}, \ldots, \frac{L_{N}}{c_{N}}\right]^{\top} \cdot \mathbf{e}_{j},
$$

where the permutation vector $\mathbf{e} \in(\{-1,1\})^{N}$ can be any element in the set satisfying $e_{1}=1$ (see that there $2^{N-1}$ elements in this subset), while the "spectral amplitudes" corresponding to $\mathbf{e}_{j}$,

$$
\mathcal{T}_{j}=\frac{1}{2^{N-1}} \sum_{k=0}^{\lfloor N / 2\rfloor} \sum_{|\mathbf{b}|=2 k}(-1)^{k+\frac{\mathrm{b} \cdot \mathbf{e}_{j}}{2}} \gamma_{\mathbf{b}},
$$

the coefficient $\gamma_{\mathrm{b}}$ associated to each binary multi-index $[25] \mathrm{b} \in(\{0,1\})^{N}$ codifies the effect of the impedance contrast at the interfaces, and it is computed as

$$
\gamma_{\mathrm{b}}=\frac{1}{2}\left(Z^{\mathrm{f}(\mathrm{b})}+\frac{1}{Z^{\mathrm{f}(\mathrm{b})}}\right)
$$

the $i$-th layer impedance is $Z_{i}=\sqrt{\rho_{i} a_{i}}$, and the map $\mathrm{f}:(\{0,1\})^{N} \rightarrow(\{-1,0,1\})^{N}$ takes the multi-index $b$, entry-wise, to the multi-index $f(b)$ defined as:

- if $\mathrm{b}_{i}=0$, then $\mathrm{f}\left(\mathrm{b}_{i}\right)=0$,

- if $\mathrm{b}_{i}=1$ and the previous value assigned by $\mathrm{f}$ to the prior 1 -entry in $\mathrm{b}$ was -1 , then $\mathrm{f}\left(\mathrm{b}_{i}\right)=1$, else $\mathrm{f}\left(\mathrm{b}_{i}\right)=-1$. 
Notice that $\gamma_{\mathrm{b}} \geq 1$ always.

Moreover, the amplitudes must meet the following condition:

$$
\sum_{k=1}^{2^{N-1}} \mathcal{T}_{k}=1
$$

In light of eq. (7), we can say that eq. (8) defines the dispersion relation for any variable-cross-section modular rod. Such a conclusion also holds for periodic laminate, as the governing equations are the same after re-branding some physical parameters.

3.1. Verifications. In order to verify eq. (8), 9 cases with different cross-sections and materials in the unit cell are studied. These 10 cases are borrowed from previous work by Kaklamanos and colleagues [26] who used Kiknet [27] profiles to analyze the transfer function in layered sites; the physical quantities are adapted to represent rod properties instead of geotechnical survey datasets. Bear in mind that these values are not intended to represent realistic devices, they are just being used for the sake of numerical verification of eq. (8).

Tthe solution provided by the analytical solution is compared to the one obtained from multiplying propagating matrices (following the customary numerical implementation used in Seismology [8]). Find in the following pages the images of two relevant scenarios whose details are presented in Table 1 and Table 2. Results for the other 7 profiles can be found in the Appendix.

TABLE 1. Information concerning the representative cell for verification in Figure 2 and Figure 3.

\begin{tabular}{|c|c|c|c|}
\hline Layer\# & $E A\left[\mathrm{kPa} \cdot \mathrm{m}^{2}\right]$ & $c[\mathrm{~m} / \mathrm{s}]$ & $L[\mathrm{~cm}]$ \\
\hline 1 & 225. & 150. & 4. \\
2 & 1650. & 1100. & 12. \\
3 & 2925. & 1950. & 30. \\
4 & 3885. & 2590. & 32. \\
\hline
\end{tabular}

TABLE 2. Information concerning the representative cell for verification in Figure 4 and Figure 5.

\begin{tabular}{|c|c|c|c|}
\hline Layer\# & $E A\left[\mathrm{kPa} \cdot \mathrm{m}^{2}\right]$ & $c[\mathrm{~m} / \mathrm{s}]$ & $L[\mathrm{~cm}]$ \\
\hline 1 & 225. & 150. & 4. \\
2 & 420. & 280. & 6. \\
3 & 420. & 280. & 10. \\
4 & 1020. & 680. & 14. \\
5 & 1350. & 900. & 16. \\
\hline
\end{tabular}



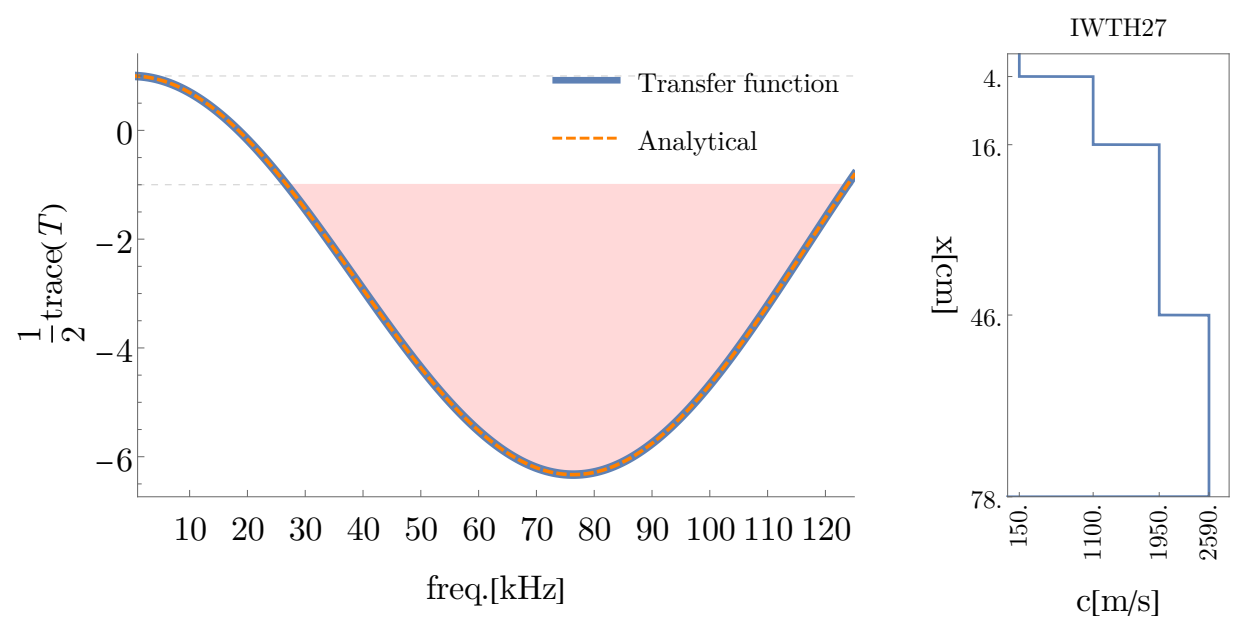

Figure 2. Half-trace function (left panel) for a rod having properties (right panel) that mirror Kik-Net site IWTH27 (shaded region in left panel corresponds to bandgap).
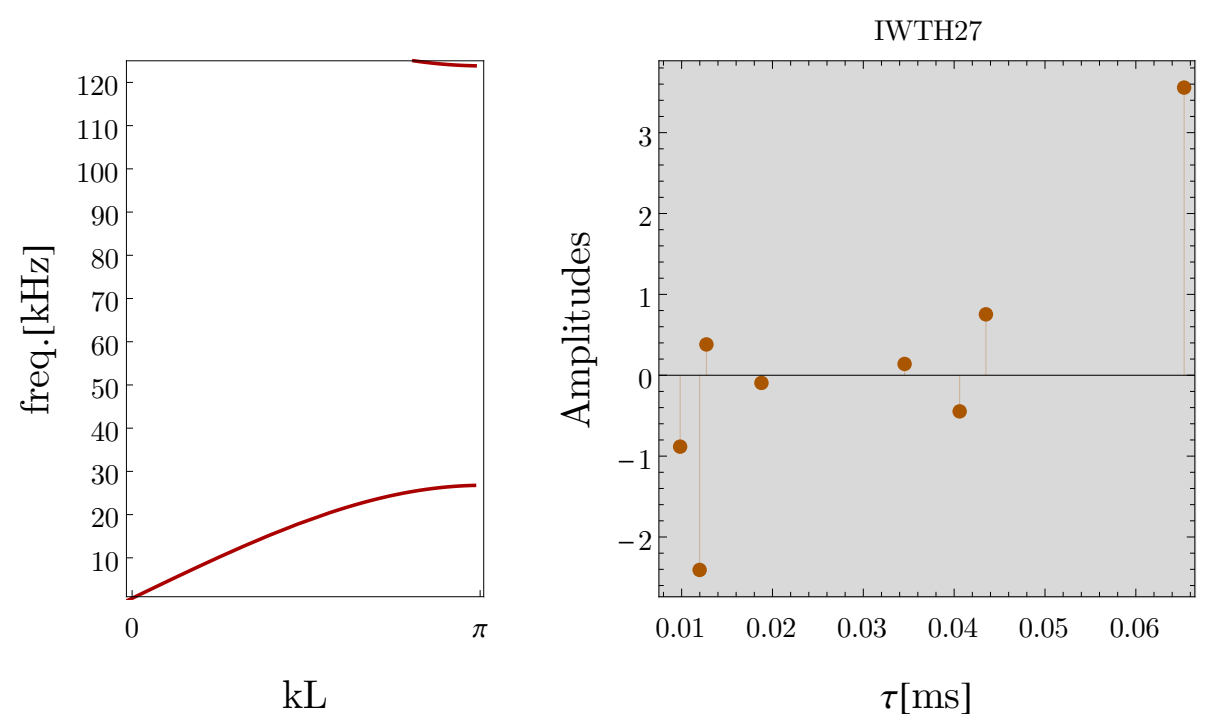

Figure 3. Dispersion diagram (left panel) and spectrum of halftrace (right panel) of rod having properties (right panel) that mirror Kik-Net site IWTH27. 

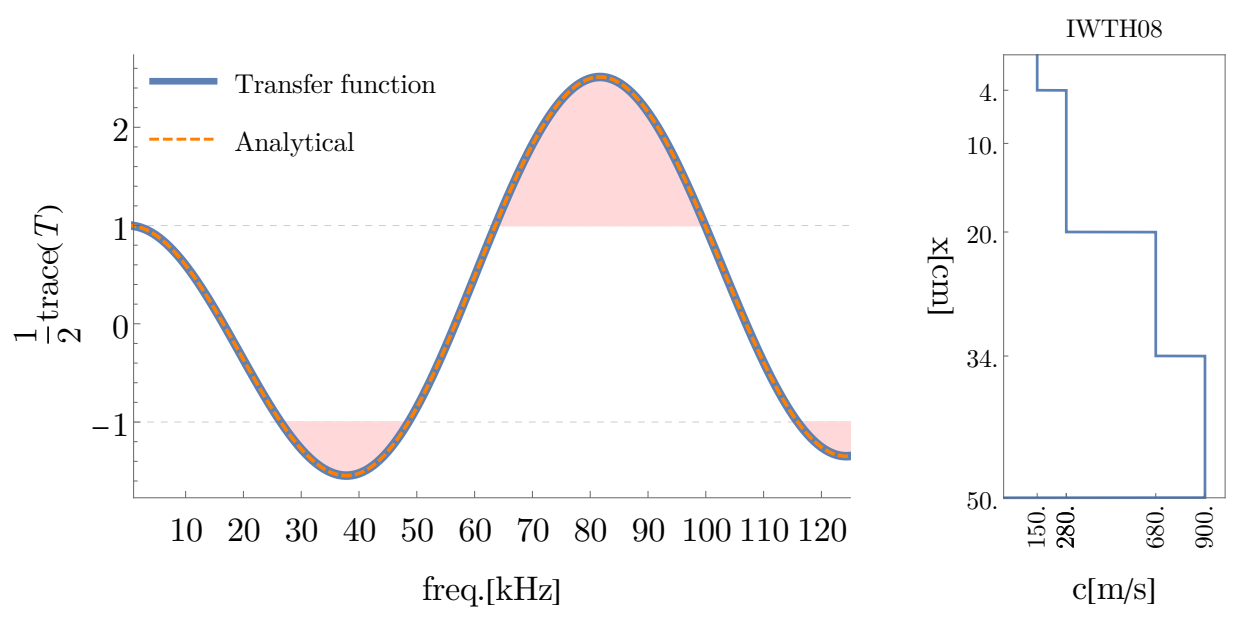

FiguRe 4. Half-trace function (left panel) for a rod having properties (right panel) that mirror Kik-Net site IWTH08 (shaded region in left panel corresponds to bandgap).
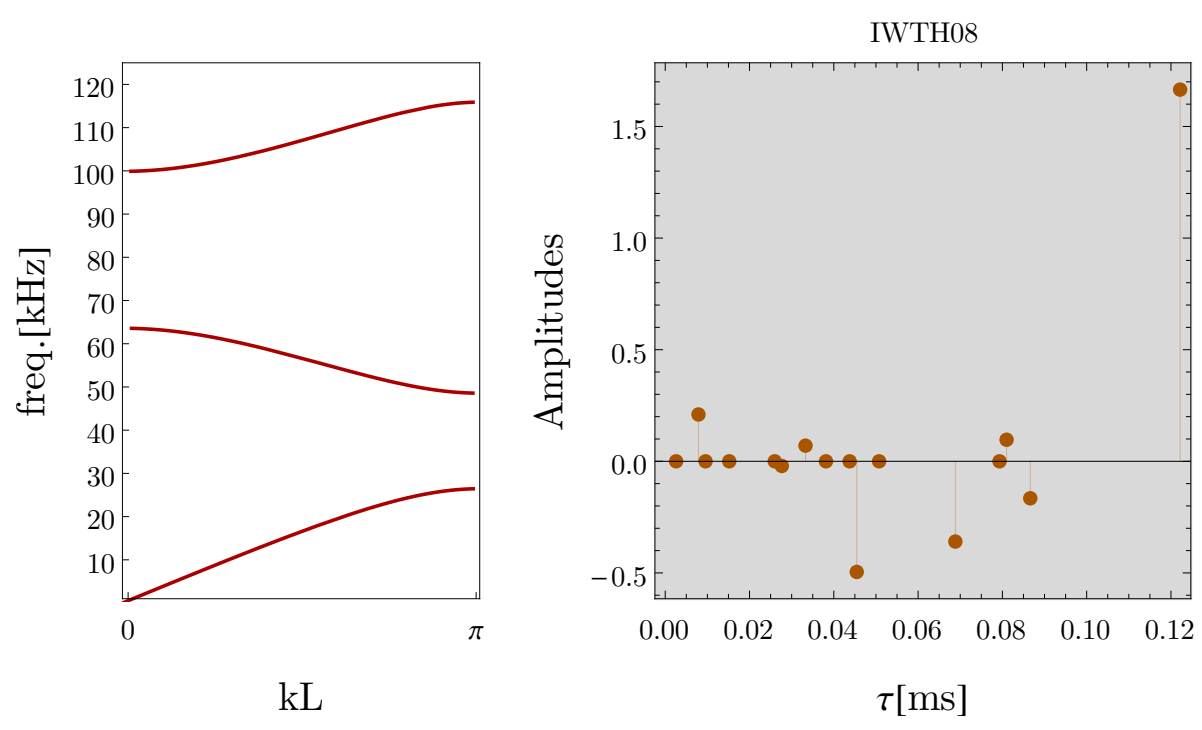

Figure 5. Dispersion diagram (left panel) and spectrum of halftrace (right panel) of rod having properties (right panel) that mirror Kik-Net site IWTH08. 
Let us discuss the previous results briefly, beyond the fact that the direct evaluation of eq. (8) yields exactly the same evolution as the numerical matrix multiplication: Figure 2 and Figure 3 correspond to a situation where there are 4 layers, hence only $2^{3}=8$ harmonics, and there are two of them whose amplitude is well above the rest (greater than 2 actually), one at the beginning and the other at the end of the spectrum, see Figure 3. See that these two harmonics are "spectrally separated", one of them can be considered as a "quick" harmonic (it oscillates relatively fast) and the other one as a "slow one" (it oscillates much slower), so they do not balk each other. Figure 4 and Figure 5, conversely, put in display an scenario where the harmonics do not interfere constructively so as to widen the first bandgap: there are a few of them, out of a total of $2^{4}=16$, that have tiny amplitude, while the rest, except for the quickest one, do not have much entity either, none of them above 0.5 in absolute value. Greater amplitudes are attained at the middle of the spectrum while the magnitude of the quick and slow harmonics is perceptibly different, see Figure 5, contrariwise to Figure 3.

Without appealing to the spectrum it would have been arduous to understand why two layer profiles that, at first, could appear to resemble each other (see left panels in both Figure 5 and Figure 3, both profiles are monotonously increasing in stiffness and the increments appear of relative magnitude), yield so different results. The information conveyed in the left panels of Figure 5 and Figure 3 evinces deep structural differences between them which were not obvious before.

The two examples in the next section will further substantiate how the dispersion relation can be tuned attending at its spectral properties. Furthermore, the harmonics will be interpreted in geometrical terms using the approach presented in [16], and design results presented in [18] will be revisited.

\section{LEVERAGing SPECTRAL PROPERTIES OF THE TRACE FOR DESign PURPOSES: WIDENING THE FIRST GAP}

4.1. Combining the knowledge as to the discrete spectrum with other recent work (universal structure). The recent work on the universal representation of the dispersion relation over a torus $[12,18,16]$ has provided a novel way to understand the design of laminated structures for 1D wave propagation.

Taking advantage of the generic form of the half-trace function $\eta(\omega)$, it was established that $|\eta(\omega)|$ can be represented over a N-dimensional torus, $\mathbb{T}^{N}$, where each coordinate of its surface was parametrized by a variables $\zeta \in \mathbb{T}^{N}, \zeta_{i} \in \mathbb{R}$ $\bmod \pi$ for $i=1 \ldots N$ (Section 4.1).

Furthermore, in this representation, the surface of the torus is divided into two regions which are independent of the the widths of the phases of the laminate yet their relative size depends on the mechanical properties of the phases (density and stiffness). Thus, the region $\mathbb{D}^{N} \subset \mathbb{T}^{N}$, defined by $|\eta|>1$, characterizes all the possible bandgaps in any dispersion relation, given a set of material properties for the phases. Thus, the torus surface encapsulates the whole set of possible dispersion relations for a multi-phase laminate, once the materials and a specific layer ordering has been decided. For any particular dispersion relation also the relative widths of the phases have to be specified, what translates onto the torus as the slope of the flow lines that traverse its surface. The discrete spectrum that we have introduced has a direct translation to the toroidal representation: the $2^{N-1}$ harmonics are the 

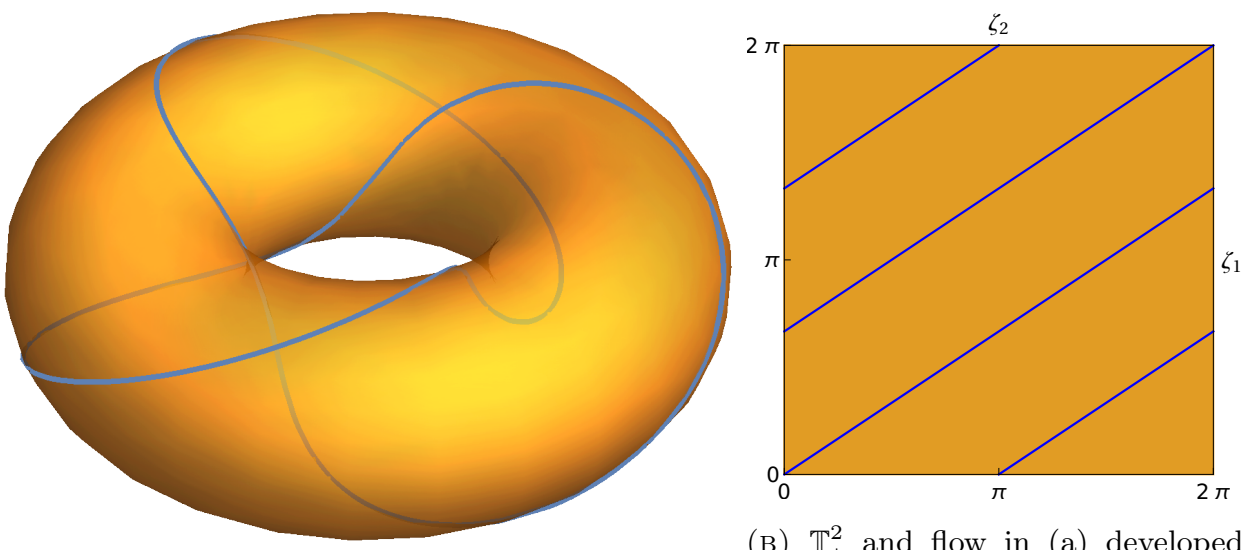

(B) $\mathbb{T}_{*}^{2}$ and flow in (a) developed over a $2 \pi \times 2 \pi$ square.

(A) Toroidal surface $\mathbb{T}_{*}^{2}$ and (periodic) linear flow winding over.

Figure 6. Representation on $\mathbb{T}^{3}$ of the dispersion relation.

elementary constituents that, when superimposed, give rise to $\mathbb{D}^{N}$ and its particular shape.

Nevertheless, except for the simple case $N=2$, the morphism between bandgaps and the portion $\mathbb{D}^{N}$ of the surface does not provide direct information on how to widen the potential gap (e.g. how to maximimize $\mathbb{D}^{N}$ within $\mathbb{T}^{N}$ or how to set up an optimal flow to intersect $\mathbb{D}^{N}$ in the most advantageous way) nor how to choose the relative widths of the phases in the representative cell.

The spectral approach does provide a general qualitative answer: widths must be chosen to augment the gap between fast and slow harmonics, while the material properties must be such that the slow harmonics are of large negative magnitude and the quick harmonics present large positive ones.

In the following two basic examples $(N=2$ and $N=3$, also considered in [18]) are explored, and the possibility of extending the design approach beyond these simple cases is pondered.

4.2. 2-layer system. The simplest configuration is made by only two phases. The corresponding half-trace function is

$$
\eta(\omega)=\cos \left(\zeta_{1}\right) \cos \left(\zeta_{2}\right)-\gamma \sin \left(\zeta_{1}\right) \sin \left(\zeta_{2}\right),
$$

where the identification $\zeta_{1}:=\omega L_{1} / c_{1}, \zeta_{2}:=\omega L_{2} / c_{2}$ is used later, and

$$
\gamma=\frac{1}{2}\left[\frac{\sqrt{\rho_{1} a_{1}}}{\sqrt{\rho_{2} a_{2}}}+\frac{\sqrt{\rho_{2} a_{2}}}{\sqrt{\rho_{1} a_{1}}}\right] .
$$

Equation (13) is so simple that it could be simplified along the lines of eq. (8) (just resorting to the cosine-of-sum formula), but let us use the multi-index formulae. eqs. (9) and (10) for the sake of illustration. 
Since $N=2$, there can be only two multi-indices of even degree:

$$
\begin{aligned}
& \mathrm{b}=(0,0) \rightarrow \mathrm{f}(\mathrm{b})=(0,0) \rightarrow C_{(0,0)}=\frac{1}{2}(1+1)=1, \\
& \mathrm{~b}=(1,1) \rightarrow \mathrm{f}(\mathrm{b})=(1,-1) \rightarrow C_{(1,1)}=\frac{1}{2}\left(\frac{\sqrt{\rho_{1} a_{1}}}{\sqrt{\rho_{2} a_{2}}}+\frac{\sqrt{\rho_{2} a_{2}}}{\sqrt{\rho_{1} a_{1}}}\right) .
\end{aligned}
$$

Likewise, the multi-index e takes only $2^{N-1}=2$ values, so the spectral periods, according to eq. (9), are

$$
\begin{gathered}
\mathbf{e}=(1,1) \rightarrow \tau_{1}=\frac{L_{1}}{c_{1}}+\frac{L_{2}}{c_{2}}, \\
\mathbf{e}=(1,-1) \rightarrow \tau_{2}=\frac{L_{1}}{c_{1}}-\frac{L_{2}}{c_{2}},
\end{gathered}
$$

while the spectral amplitudes, following eq. (10),

$$
\begin{aligned}
& \mathbf{e}=(1,1) \rightarrow \mathcal{T}_{1}=\frac{1}{2}\left((-1)^{0+\frac{1}{2}(0,0) \cdot(1,1)} 1+(-1)^{1+\frac{1}{2}(1,1) \cdot(1,1)} \gamma\right)=\frac{1+\gamma}{2} \\
& \mathbf{e}=(1,-1) \rightarrow \mathcal{T}_{2}=\frac{1}{2}\left((-1)^{0+\frac{1}{2}(0,0) \cdot(1,-1)} 1+(-1)^{1+\frac{1}{2}(1,1) \cdot(1,-1)} \gamma\right)=\frac{1-\gamma}{2}
\end{aligned}
$$

So the half-trace function is

$$
\eta(\omega)=\frac{1}{2} \tan (\boldsymbol{T}(0, L))=\frac{1+\gamma}{2} \cos \left(\zeta_{1}+\zeta_{2}\right)+\frac{1-\gamma}{2} \cos \left(\zeta_{1}-\zeta_{2}\right),
$$

let us show the physical parameters explicitly,

$$
\begin{aligned}
& =\frac{1}{2}\left(1+\frac{1}{2}\left[\frac{\sqrt{\rho_{1} a_{1}}}{\sqrt{\rho_{2} a_{2}}}+\frac{\sqrt{\rho_{2} a_{2}}}{\sqrt{\rho_{1} a_{1}}}\right]\right) \cos \left(\left[\frac{L_{1}}{c_{1}}+\frac{L_{2}}{c_{2}}\right] \omega\right) \text { (first harmonic) } \\
& +\frac{1}{2}\left(1-\frac{1}{2}\left[\frac{\sqrt{\rho_{1} a_{1}}}{\sqrt{\rho_{2} a_{2}}}+\frac{\sqrt{\rho_{2} a_{2}}}{\sqrt{\rho_{1} a_{1}}}\right]\right) \cos \left(\left[\frac{L_{1}}{c_{1}}-\frac{L_{2}}{c_{2}}\right] \omega\right) \text { (second harmonic) }
\end{aligned}
$$

Hence, the first harmonic is the "quick harmonic" (greater spectral period) while the second one is the "slow" one. Bear in mind that the labeling as "first" and subsequent ordinals do not hold the usual meaning they carry in Fourier Analysis (e.g., faster oscillations as the ordinal increases), it just refers to the permutation vectorm $\boldsymbol{e}$ they are associated to. In order to maximize the first bandgap, the quick harmonic must attain the maximum negative amplitude while the other one, the maximum positive one. Notice how the first harmonic remains constant over lines $\zeta_{1}+\zeta_{2}=c \in \mathbb{R}$, while the second does so $\zeta_{1}-\zeta_{2}=c$.

See that $\mathcal{T}_{1} \propto \gamma$ while $\mathcal{T}_{2} \propto-\gamma$, thus to make the first amplitude more positive and the second one more negative, one just has to keep increasing $\gamma$. It is therefore better to ask, given a certain $\gamma$ (e.g., fixing the material of the layers), what is the most efficient way to distribute the layer thicknesses? If one wants to make the second harmonic as slow as possible, the most logical option is to make it not to oscillate at all, so $\tau_{2}=0 \rightarrow h_{1} / c_{1}=h_{2} / c_{2}$ achieves that. Let us use the representation on the surface of a torus, as suggested in [12], to gain extra insight. Instead of using the reduced torus $\mathbb{T}^{2}$, where the variables are defined $\bmod \pi$, let us use the "extended torus" $\mathbb{T}_{*}^{2}$, such that $\zeta_{1}, \zeta_{2} \in \mathbb{R} \bmod 2 \pi$. 


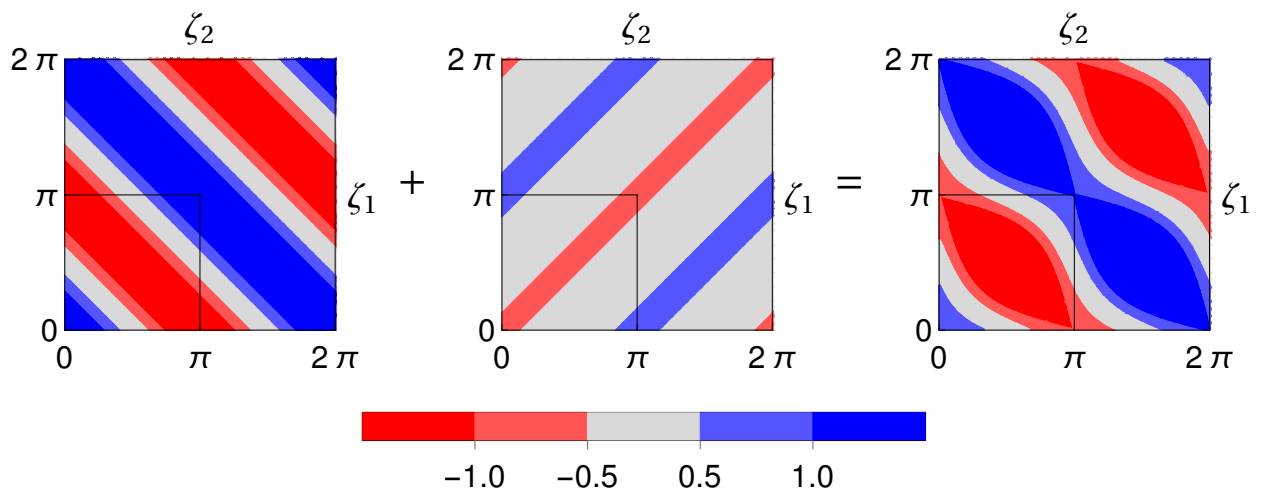

FiguRE 7. Representation over $\mathbb{T}_{*}^{2}$ of, from left to right: first harmonic, second harmonic and total half-trace function (sum of harmonics). Using value $\gamma=2.125$ for the plots. The bottom-left quadrant delimitates the reduced torus $\mathbb{T}^{2}$.

The physical values used to visualize results (these values yield $\gamma=2.125$ ) are:

$$
\begin{aligned}
& \rho_{1}=1500 \mathrm{~kg} / \mathrm{m}^{3}, c_{1}=100 \mathrm{~m} / \mathrm{s}, L_{1}=75 . \mathrm{cm}, \\
& \rho_{2}=1500 \mathrm{~kg} / \mathrm{m}^{3}, c_{2}=400 . \mathrm{m} / \mathrm{s}, L_{2}=300 . \mathrm{cm} .
\end{aligned}
$$

In Figure 7, the toroidal surface as square is developed over a square, both side lengths being $2 \pi$, where opposite flanks are identified and the four vertices represent the same point (origin for $\boldsymbol{\zeta}$ ).

The left-most square corresponds to the first harmonic, whose pattern is of course a cosine function supported by the diagonal $\zeta_{1}=\zeta_{2}$, scaled by the corresponding amplitude $\mathcal{T}_{1}$. We can think of these patters as the building blocks of $\mathbb{D}^{2}$. The middle square represents the second harmonic, a cosine function supported by the diagonal $\zeta_{1}+\zeta_{2}=1$ and scaled by $\mathcal{T}_{2}$. Finally, the third square is the "sum" of the two, which now yields similar diagrams as those presented in [12]. See that the first quadrant is the only one relevant: in practice, only the absolute value of the half-trace function is relevant to characterize bandgaps, thus the information in the other three quadrants would be redundant.

The bandgap region $\mathbb{D}^{2}$ is the red blob in the first quadrant, it codifies all possible dispersion relations. The missing piece to set a specific dispersion relation up is defining the intersections (frequencies) for which the linear flow enters and exits this region.

Having the representation over the torus at hand, let us ponder on the meaning of the choice $h_{1} / c_{1}=h_{2} / c_{2}$, which renders the slow harmonic constant.

If our intention is to maximize the first gap, it seems logical to stick to the red region of the second harmonic that crosses the origin for as long as possible, what is attained precisely when we stick to the diagonal $\zeta_{1}=\zeta_{2} \rightarrow h_{1} / c_{1}=h_{2} / c_{2}$, precisely. So making the second harmonic as slow as possible amounts to fixing the flow to follow a $45 \mathrm{deg}$ inclination angle, along the main diagonal of the square. Moving along the diagonal does not maximize the distance between intersections with the red region of the first harmonic (leftmost square), but deviating from the diagonal appears to produce a counterproductive effect. 

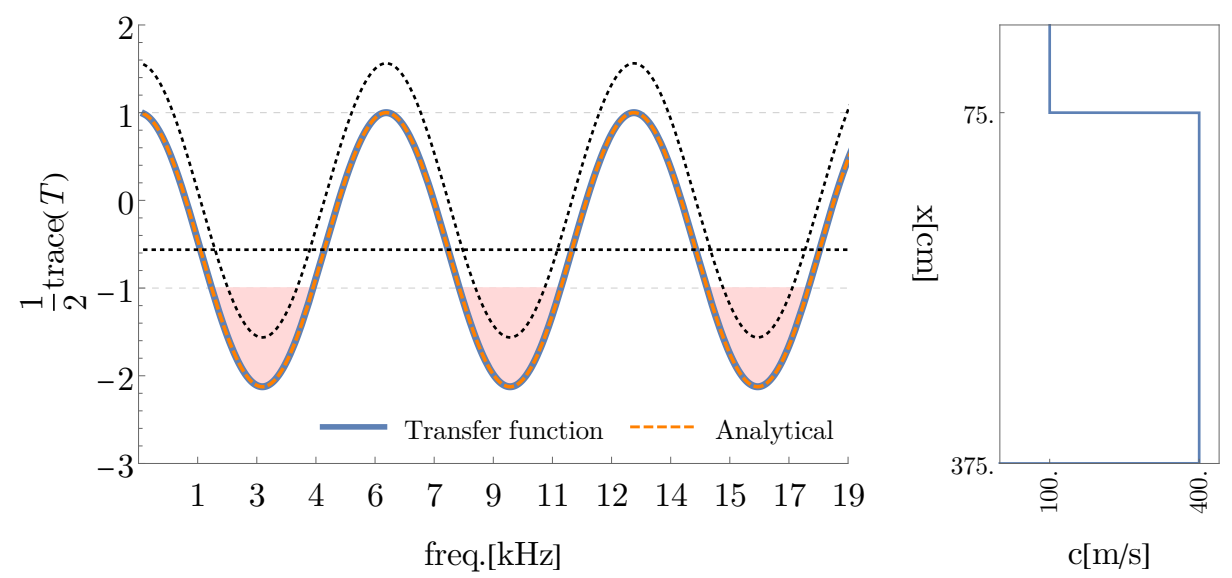

Figure 8. Profile designed (right panel) and half-trace function (left panel) with corresponding shaded regions corresponding to bandgaps; the slow harmonic corresponds to the dashed horizontal line, the fast one to the dashed oscillating one.

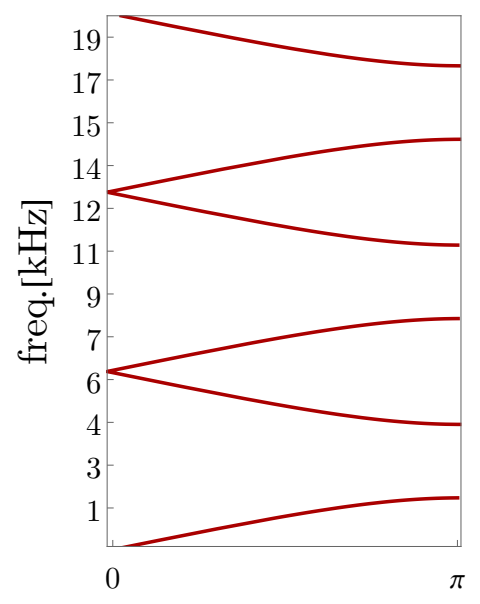

$\mathrm{kL}$

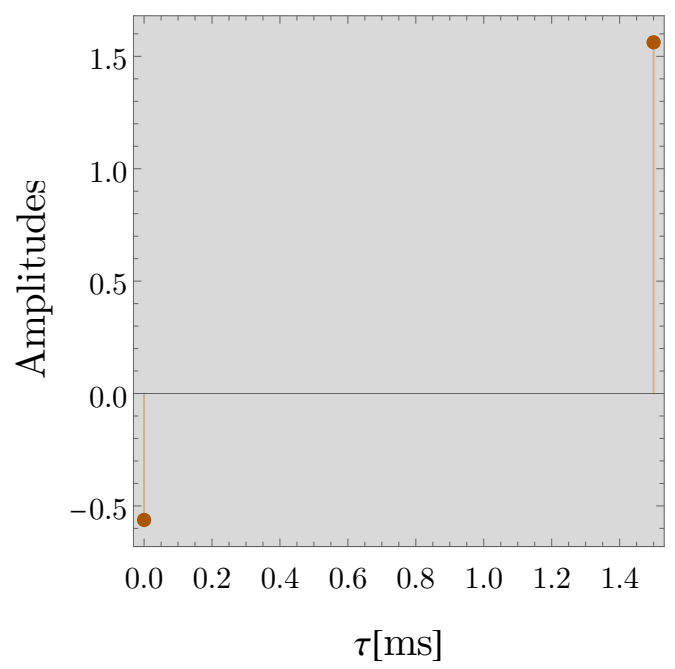

FiguRE 9. Dispersion relation diagram (left panel) and one-sided spectrum (right panel) of the design in Figure 8.

This conclusion, that the first gap is optimized whenthe flow sticks to the main diagonal of the square, was also attained in [18] by purely algebraic means. In 
that article, not only the relation between wave relative widths was found, but also it was concluded that the maximum possible was attained when $c_{1}=c_{2}$ (so also $L_{1} / c_{1}=L_{2} / c_{2}$ implies $L_{1}=L_{2}=L / 2$ ) the impedance constant would arise solely from the mismatch of densities. In practice, material science establishes that wave velocity and density are strongly correlated, as both of this macro-properties arise from the micromechanical structure of the matter.

The spectral approach illuminates what the essential building blocks of the dispersion relation are and, in tandem with the representation over the torus, allowz for a neat assessment of the possibilities from a design standpoint.

4.3. 3-layer system. In this case, $N=3$, we have the following non-trivial possible $b$ values (apart from the trivial one), which are mapped by $f$ as

$$
\begin{aligned}
& \mathrm{b}=(1,1,0) \rightarrow \mathrm{f}(\mathrm{b})=(1,-1,0) \rightarrow \gamma_{(1,2)}=\frac{1}{2}\left(\frac{\sqrt{\rho_{1} a_{1}}}{\sqrt{\rho_{2} a_{2}}}+\frac{\sqrt{\rho_{2} a_{2}}}{\sqrt{\rho_{1} a_{1}}}\right), \\
& \mathrm{b}=(1,0,1) \rightarrow \mathrm{f}(\mathrm{b})=(1,0,-1) . \rightarrow \gamma_{(1,3)}=\frac{1}{2}\left(\frac{\sqrt{\rho_{1} a_{1}}}{\sqrt{\rho_{3} a_{3}}}+\frac{\sqrt{\rho_{3} a_{3}}}{\sqrt{\rho_{1} a_{1}}}\right), \\
& \mathrm{b}=(0,1,1) \rightarrow \mathrm{f}(\mathrm{b})=(0,1,-1) \rightarrow \gamma_{(2,3)}=\frac{1}{2}\left(\frac{\sqrt{\rho_{2} a_{2}}}{\sqrt{\rho_{3} a_{3}}}+\frac{\sqrt{\rho_{3} a_{3}}}{\sqrt{\rho_{2} a_{2}}}\right),
\end{aligned}
$$

where the simplified notation $\gamma_{(n, m)}$ indicate that the impedance contrast is formed with the properties of the $n$-th and the $m$-th layer.

Likewise, the multi-index $\mathbf{e} \in(\{-1,1\})^{N}$ yields the periods, and also the amplitudes in tandem with the corresponding $f(b)$ :

$$
\mathbf{e}_{1}=(1,1,1) \rightarrow \tau_{1}=\frac{L_{1}}{c_{1}}+\frac{L_{2}}{c_{2}}+\frac{L_{3}}{c_{3}} \text { and } 4 \mathcal{T}_{1}=1+\gamma_{(1,2)}+\gamma_{(1,3)}+\gamma_{(2,3)},
$$

$$
\mathbf{e}_{2}=(1,-1,1) \rightarrow \tau_{2}=\frac{L_{1}}{c_{1}}-\frac{L_{2}}{c_{2}}+\frac{L_{3}}{c_{3}} \text { and } 4 \mathcal{T}_{2}=1-\gamma_{(1,2)}+\gamma_{(1,3)}-\gamma_{(2,3)},
$$

$$
\mathbf{e}_{3}=(1,1,-1) \rightarrow \tau_{3}=\frac{L_{1}}{c_{1}}+\frac{L_{2}}{c_{2}}-\frac{L_{3}}{c_{3}} \text { and } 4 \mathcal{T}_{3}=1+\gamma_{(1,2)}-\gamma_{(1,3)}-\gamma_{(2,3)},
$$

$$
\mathbf{e}_{4}=(1,-1,-1) \rightarrow \tau_{4}=\frac{L_{1}}{c_{1}}-\frac{L_{2}}{c_{2}}-\frac{L_{3}}{c_{3}} \text { and } 4 \mathcal{T}_{4}=1-\gamma_{(1,2)}-\gamma_{(1,3)}+\gamma_{(2,3)},
$$

see how, indeed, $\mathcal{T}_{1}+\mathcal{T}_{2}+\mathcal{T}_{3}+\mathcal{T}_{4}=1$. If the first harmonic oscillates much faster than the others, it will cause the half-trace to go well beyond -1 before the overall amplitude is affected by the other oscillatory terms.

Therefore, let us re-state the design approach as maximizing simultaneously $\mathcal{T}_{1}$ and $\tau_{1}$, the latter with respect to all the others $\tau_{i}$ for all $i>1$.

Moreover, in this case, one can make the potentially-slowest harmonic, the one corresponding to $\zeta_{1}-\zeta_{2}-\zeta_{3}=\omega\left(L_{1} / c_{1}-L_{2} / c_{2}-L_{3} / c_{3}\right)$, not to oscillate at all if lengths and velocities are chosen such that $\left(L_{1} / c_{1}-L_{2} / c_{2}-L_{3} / c_{3}\right) \approx 0$. If the corresponding amplitude is negative, it would mean that its effect reinforces the bandgap generated by the oscillation of the quick harmonic.

Finally, the two intermediate harmonics can be made to cross zero (and add no 
amplitude in either way) at the same time that the quick harmonic reaches its first peak, by sensibly choosing the relation between lengths and wave velocity.

For the sake of clarity, let us show in all detail how the global half-trace looks like:

$$
\begin{aligned}
& \eta(\omega)=\frac{1}{2} \tan (\boldsymbol{T}(0, L))= \\
& \frac{1}{4}\left(1+\gamma_{(1,2)}+\gamma_{(1,3)}+\gamma_{(3,2)}\right) \cos \left(\zeta_{1}+\zeta_{2}+\zeta_{3}\right) \\
& +\frac{1}{4}\left(1-\gamma_{(1,2)}+\gamma_{(1,3)}-\gamma_{(3,2)}\right) \cos \left(\zeta_{1}-\zeta_{2}+\zeta_{3}\right) \\
& +\frac{1}{4}\left(1+\gamma_{(1,2)}-\gamma_{(1,3)}-\gamma_{(3,2)}\right) \cos \left(\zeta_{1}+\zeta_{2}-\zeta_{3}\right) \\
& +\frac{1}{4}\left(1-\gamma_{(1,2)}-\gamma_{(1,3)}+\gamma_{(3,2)}\right) \cos \left(\zeta_{1}-\zeta_{2}-\zeta_{3}\right) \\
& =\frac{1}{4}\left(1+\frac{1}{2}\left[\frac{\sqrt{\rho_{1} a_{1}}}{\sqrt{\rho_{2} a_{2}}}+\frac{\sqrt{\rho_{2} a_{2}}}{\sqrt{\rho_{1} a_{1}}}\right]+\frac{1}{2}\left[\frac{\sqrt{\rho_{1} a_{1}}}{\sqrt{\rho_{3} a_{3}}}+\frac{\sqrt{\rho_{3} a_{3}}}{\sqrt{\rho_{1} a_{1}}}\right]+\frac{1}{2}\left[\frac{\sqrt{\rho_{2} a_{2}}}{\sqrt{\rho_{3} a_{3}}}+\frac{\sqrt{\rho_{3} a_{3}}}{\sqrt{\rho_{2} a_{2}}}\right]\right) \\
& \cos \left(\left[\frac{L_{1}}{c_{1}}+\frac{L_{2}}{c_{2}}+\frac{L_{3}}{c_{3}}\right] \omega\right) \text { (first harmonic) } \\
& +\frac{1}{4}\left(1-\frac{1}{2}\left[\frac{\sqrt{\rho_{1} a_{1}}}{\sqrt{\rho_{2} a_{2}}}+\frac{\sqrt{\rho_{2} a_{2}}}{\sqrt{\rho_{1} a_{1}}}\right]+\frac{1}{2}\left[\frac{\sqrt{\rho_{1} a_{1}}}{\sqrt{\rho_{3} a_{3}}}+\frac{\sqrt{\rho_{3} a_{3}}}{\sqrt{\rho_{1} a_{1}}}\right]-\frac{1}{2}\left[\frac{\sqrt{\rho_{2} a_{2}}}{\sqrt{\rho_{3} a_{3}}}+\frac{\sqrt{\rho_{3} a_{3}}}{\sqrt{\rho_{2} a_{2}}}\right]\right) \\
& \cos \left(\left[\frac{L_{1}}{c_{1}}-\frac{L_{2}}{c_{2}}+\frac{L_{3}}{c_{3}}\right] \omega\right) \text { (second harmonic) } \\
& +\frac{1}{4}\left(1+\frac{1}{2}\left[\frac{\sqrt{\rho_{1} a_{1}}}{\sqrt{\rho_{2} a_{2}}}+\frac{\sqrt{\rho_{2} a_{2}}}{\sqrt{\rho_{1} a_{1}}}\right]-\frac{1}{2}\left[\frac{\sqrt{\rho_{1} a_{1}}}{\sqrt{\rho_{3} a_{3}}}+\frac{\sqrt{\rho_{3} a_{3}}}{\sqrt{\rho_{1} a_{1}}}\right]-\frac{1}{2}\left[\frac{\sqrt{\rho_{2} a_{2}}}{\sqrt{\rho_{3} a_{3}}}+\frac{\sqrt{\rho_{3} a_{3}}}{\sqrt{\rho_{2} a_{2}}}\right]\right) \\
& \cos \left(\left[\frac{L_{1}}{c_{1}}+\frac{L_{2}}{c_{2}}-\frac{L_{3}}{c_{3}}\right] \omega\right) \text { (third harmonic) } \\
& +\frac{1}{4}\left(1-\frac{1}{2}\left[\frac{\sqrt{\rho_{1} a_{1}}}{\sqrt{\rho_{2} a_{2}}}+\frac{\sqrt{\rho_{2} a_{2}}}{\sqrt{\rho_{1} a_{1}}}\right]-\frac{1}{2}\left[\frac{\sqrt{\rho_{1} a_{1}}}{\sqrt{\rho_{3} a_{3}}}+\frac{\sqrt{\rho_{3} a_{3}}}{\sqrt{\rho_{1} a_{1}}}\right]+\frac{1}{2}\left[\frac{\sqrt{\rho_{2} a_{2}}}{\sqrt{\rho_{3} a_{3}}}+\frac{\sqrt{\rho_{3} a_{3}}}{\sqrt{\rho_{2} a_{2}}}\right]\right) \\
& \cos \left(\left[\frac{L_{1}}{c_{1}}-\frac{L_{2}}{c_{2}}-\frac{L_{3}}{c_{3}}\right] \omega\right) \cdot(\text { fourth harmonic })
\end{aligned}
$$

The material properties used for this design example are:

$$
\begin{aligned}
\rho_{1} & =1500 . \mathrm{kg} / \mathrm{m}^{3}, c_{1}=100 . \mathrm{m} / \mathrm{s}, L_{1}=30 . \mathrm{cm}, \\
\rho_{2} & =1500 . \mathrm{kg} / \mathrm{m}^{3}, c_{2}=300 . \mathrm{m} / \mathrm{s}, L_{2}=45 . \mathrm{cm}, \\
\rho_{3} & =1500 . \mathrm{kg} / \mathrm{m}^{3}, c_{3}=900 . \mathrm{m} / \mathrm{s}, L_{3}=135 . \mathrm{cm} .
\end{aligned}
$$

The design proposed in Figure 10 achieves the goals that were set above. Figure 11 displays the equivalent dispersion relation in the right panel and, more importantly, the spectrum we envisioned in the left one.

Section 4.4 shows the corresponding representation on the reduced torus $\mathbb{T}^{3}$. The left images represent the harmonics individually, see how the top-left one that corresponds to the first (quick) harmonic has contour planes perpendicular to the main diagonal $\zeta_{1}=\zeta_{2}=\zeta_{3}$ while the fourth (slow) one; these two harmonics are the ones displaying the greatest amplitudes. The right cube represents the outcome of superimposing the individual harmonics, what gives rise to shapes similar to those 
obtained in [18]. An arrow traversing the main diagonal is depicted on the same image too, indicating the flow used in the design.
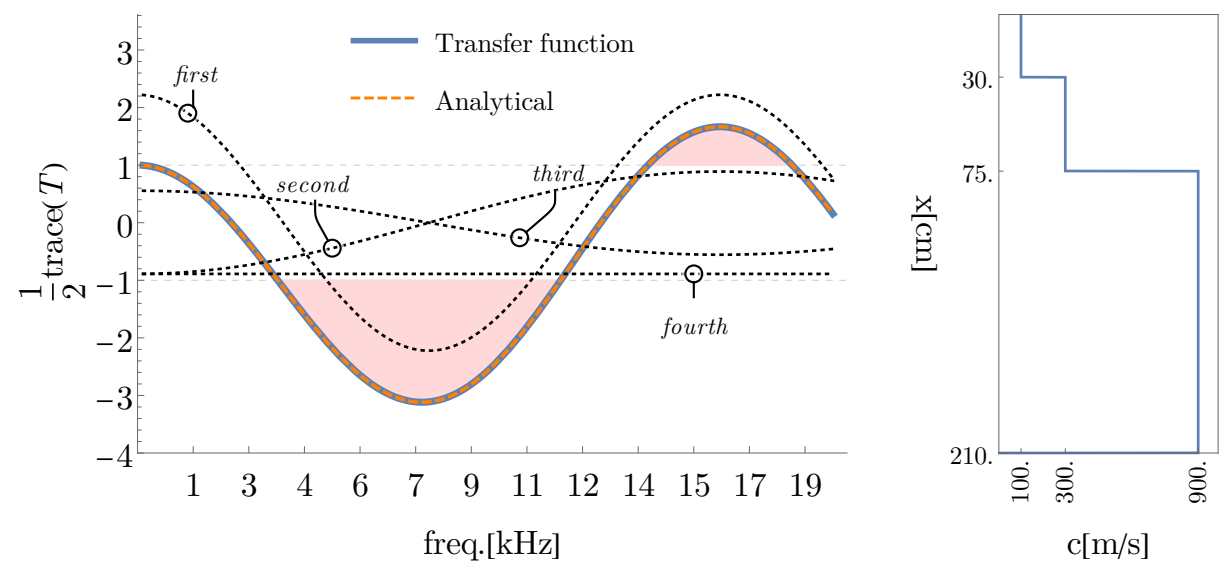

Figure 10. Profile designed (right panel) and half-trace function (left panel) with corresponding shaded regions corresponding to bandgaps; the harmonics are marked with dotted lines and called by their order (see section 4.3).
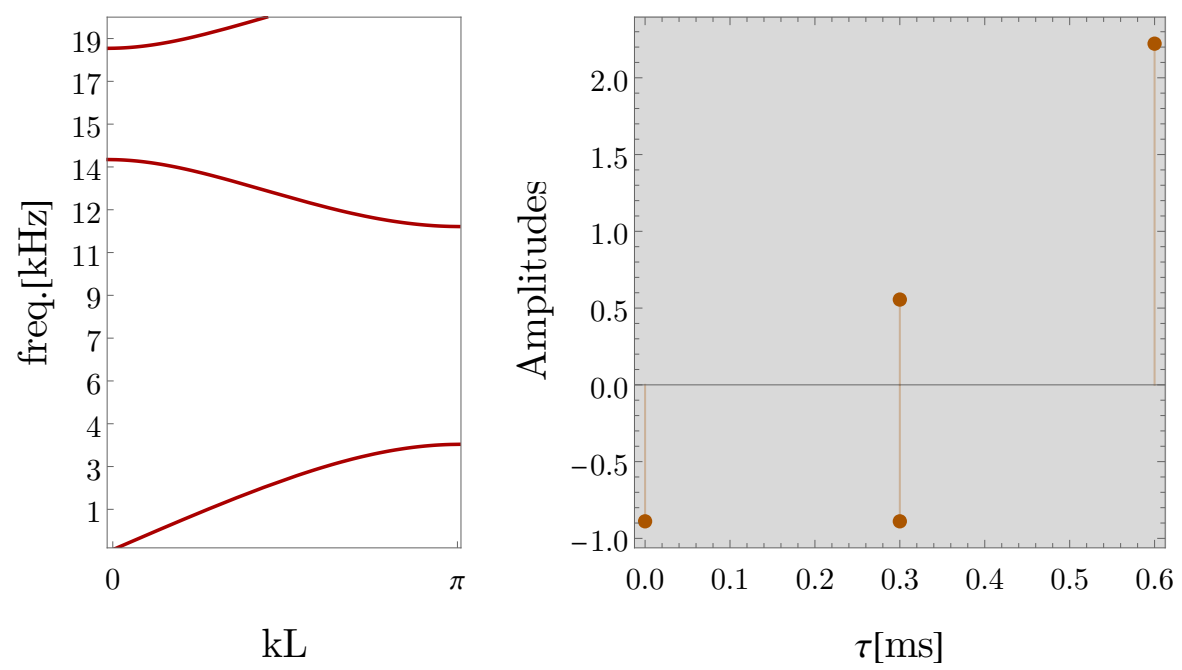

Figure 11. Dispersion relation diagram (left panel) and one-sided spectrum (right panel) of the design in Figure 11. 

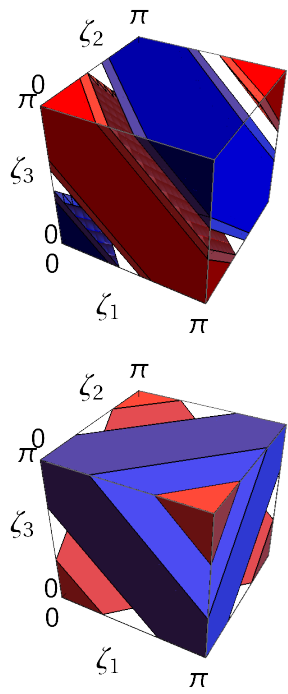

(A) Bandgap regions for each harmonic (ordered from top to bottom, from left to right)

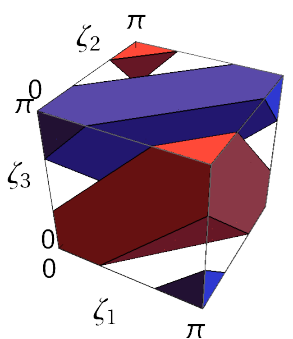

(B) Overall band-gap regions (superposition of the four harmonics). Black arrow indicates the main diagonal $\zeta_{1}=\zeta_{2}=\zeta_{3}$.

FiguRE 12. Representation on $\mathbb{T}^{3}$ of the dispersion relation.

4.4. Discussion. Looking at Figure 2, it appears that one could do even better: the approach would consist of having many "slow" harmonics with large negative amplitude and many "quick" harmonics with large positive amplitude, while making sure that the amplitude of intermediate harmonics remains relatively small. As a matter of fact, Figure 3 displays just that: out of 8 harmonics, the two lowest ones have negative amplitude much greater than the next five ones, and the eighth and last one does possess positive amplitude greater than the 5 previous ones and of the same order as the two slowest ones.

From the prior considerations one can posit that an "ideal" spectrum (in the sense of lowest-frequency-bandgap maximization) would display a "S"-shaped distribution of amplitudes: large and negative at the lowest values and large and positive at the largest ones, while intermediate harmonics' amplitudes would be much smaller. When moving to the toroidal representation, it seems the said amounts to moving along the main diagonal of $\mathbb{T}^{N}$, given a set of material constants. This has been shown for the simplest cases $N=2$ and $N=3$. It is tempting to assume that this design recipe would yield optimal results for any selection of materials (e.g. any material contrasts between layers) and for any number of layers $N$, but this remains to be formally shown. 


\section{FinAL REMARKS AND FUTURE WORK}

It has been shown that the half-trace function that governs the dispersion relation in periodic layered media possesses neat spectral properties: namely, the one-sided spectrum is made up by $2^{N-1}$ distinct harmonics, whose period is independent of the order of the layering, but whose amplitudes and phase delay do depend on the disposition of the layers. Conversely, the amplitudes are independent of the relative thicknesses of the layers whereas the periods do depend on them. It has also been shown that the phase delay between harmonics is either 0 or $\pi$ radians, and that the dispersion relation of the laminate can be constructed using combinatorics expressed by multi-indices. As mentioned repeatedly, the discrete spectrum of the half-trace not only unveils a new understanding of the dispersion relation features, it also can serve also as an innovatory design tool. Section 4 showcased how the first gap can be optimized employing spectral arguments. The spectrum of the half-trace may be the means to unveil all the design possibilities when it comes to engineering dispersion relations in complex layered media.

When it comes to future contributions in the more theoretical aspects, it seems paramount to obtain a satisfactory mathematical explanation of the underlying structure of the problem. For instance, there better be a good reason justifying the hefty presence of combinatorics (multi-indices). The answer to this and similar questions may come from Group Theory. A deeper appraisal of the mathematical structure of the phenomenon, beyond its purely theoretical interest, could provide compelling answers as to the design space possibilities. Theorems as to optimality of the lowest-frequency bandgap are sought too, an important question to be answered is: when does the flow along the main diagonal of the torus correspond to the widest-possible first bandgap?

The many branches of Physics and Engineering where the transfer matrix method finds application could benefit from the knowledge of the spectrum of the half-trace function.

In the realm of metamaterials, the dispersion relation of electromagnetic and acoustic waves can be engineered as well. In Optics, certain entries of the global propagator can be related to the reflectivity of the layering, so optimizing in terms of spectrum would also be possible there.

There are many other applications in Linear Elasticity beyond dispersion relations. Seismology applications were mentioned in Section 1, so let us delve on the two wave propagation scenarios whose transfer matrices are two-by-two matrices:

- Inclined SH-wave systems: to consider this scenario we would have to qualitatively modify one entry of the propagator matrix in order to introduce the ray parameter $p[8]$; see that the governing vector ODE, recall eq. (3), in this case is

$$
\frac{d}{d z}\left[\begin{array}{c}
\hat{u}_{y} \\
\hat{\tau}_{y z}
\end{array}\right]=\left[\begin{array}{cc}
0 & 1 / \mu \\
\omega^{2}\left(\mu p^{2}-\rho\right) & 0
\end{array}\right]\left[\begin{array}{c}
\hat{u}_{y} \\
\hat{\tau}_{y z}
\end{array}\right]=\boldsymbol{A f}(z),
$$

where $\rho$ and $\mu$ are the density and shear modulus of the material conforming the layer, and $p=\sin (\gamma) / \sqrt{\mu / \rho}$, where $\gamma$ is the inclination angle with respect to the normal at the interface. See that now $\boldsymbol{A}$ can be rank-deficient: even though still trace $(\boldsymbol{A})=0$, the determinant of the layer matrix can equal zero, unlike in the previous case or for $\mathrm{SH}$ waves impinging normally to the interfaces $(\gamma=0)$. The latter happens precisely when ray arrived in 
the layer at the critical angle. Let us also mention that the propagation of inclined electromagnetic and acoustic waves obeys a similar formalism.

- Love waves: likewise, for this kind of surface waves [8], relevant displacement and stresses can be written as

$$
\begin{aligned}
u_{y} & =l_{1}(\mathrm{k}, z, \omega) e^{\mathrm{i}(\mathrm{k} x-\omega t)}, \\
\tau_{y z} & =l_{2}(\mathrm{k}, z, \omega) e^{\mathrm{i}(\mathrm{k} x-\omega t)}, \\
\tau_{x y} & =\mathrm{ik} l_{1}(\mathrm{k}, z, \omega) e^{\mathrm{i}(\mathrm{k} x-\omega t)},
\end{aligned}
$$

where $z$ represents depth measure from the free surface, and the equivalent of eq. (3) is therefore

$$
\frac{d}{d z}\left[\begin{array}{l}
l_{1} \\
l_{2}
\end{array}\right]=\left[\begin{array}{cc}
0 & 1 / \mu \\
\mu \mathrm{k}^{2}-\rho \omega^{2} & 0
\end{array}\right]\left[\begin{array}{l}
l_{1} \\
l_{2}
\end{array}\right]=\boldsymbol{A} \boldsymbol{f}(z) .
$$

The determinant of the layer matrix goes one step beyond now: it can take any real value, including negative ones. As a consequence of this, the entries of the elementary transfer matrix for a given layer can take complex values (as they are proportional to the square root of the aforementioned determinant), what means that one would have to examine the group $S L(2, \mathbb{C})$ in lieu of $S L(2, \mathbb{R})$. Another distinct and interesting feature of considering surface waves in general is that two boundary conditions, (a) stress-free surface and (b) vanishing at infinite depth, replace periodicity conditions.

There are other applications in Seismology, inclined P-SV wave systems and surface Rayleigh waves, but require four-by-four matrices, and they seem hence more difficult to tackle.

\section{ACKNOWLEDGEMENTS}

I would like to thank Prof. J.-F. Molinari (EPFL), Prof. G. Subhash (University of Florida) and Prof. G. Shmuel (Technion) for their comments, which have greatly improved this manuscript.

\section{SUPPLEMENTARY MATERIAL}

A Mathematica notebook [28] containing the computations leading to results (including figures) shown in the text can be found in the repository named spectrum_trace in the author's GitHub page github.com/jgarciasuarez.

\section{REFERENCES}

[1] John Lekner. Light in periodically stratified media. JOSA A, 11(11):2892-2899, 1994.

[2] Louis A. Pipes. Matrix analysis of heat transfer problems. Journal of the Franklin Institute, 263(3):195-206, 1957.

[3] Mahito Kohmoto, Leo P Kadanoff, and Chao Tang. Localization problem in one dimension: Mapping and escape. Physical Review Letters, 50(23):1870, 1983.

[4] Mahito Kohmoto, Bill Sutherland, and K Iguchi. Localization of optics: Quasiperiodic media. Physical review letters, 58(23):2436, 1987.

[5] Enrique Maciá. Hierarchical description of phonon dynamics on finite fibonacci superlattices. Physical review B, 73(18):184303, 2006.

[6] David Damanik, Anton Gorodetski, and William Yessen. The fibonacci hamiltonian. Inventiones mathematicae, 206(3):629-692, 2016.

[7] Mingrong Shen and Wenwu Cao. Acoustic bandgap formation in a periodic structure with multilayer unit cells. Journal of Physics D: Applied Physics, 33(10):1150, 2000.

[8] Keiiti Aki and Paul G Richards. Quantitative seismology. University Science Books, 2002. 
[9] Ari Ben-Menahem and Sarva Jit Singh. Seismic waves and sources. Springer Science \& Business Media, 2012.

[10] Steven L Kramer. Geotechnical earthquake engineering. in Prentice-Hall international series in civil engineering and engineering mechanics. Prentice Hall Inc., Englewood Cliffs, New Jersey, 1996.

[11] Freeman Gilbert and George E Backus. Propagator matrices in elastic wave and vibration problems. Geophysics, 31(2):326-332, 1966.

[12] Gal Shmuel and Ram Band. Universality of the frequency spectrum of laminates. Journal of the Mechanics and Physics of Solids, 92:127-136, 2016.

[13] Ankit Srivastava. Metamaterial properties of periodic laminates. Journal of the Mechanics and Physics of Solids, 96:252-263, 2016.

[14] John R Willis. Negative refraction in a laminate. Journal of the Mechanics and Physics of Solids, 97:10-18, 2016.

[15] Lorenzo Morini and Massimiliano Gei. Waves in one-dimensional quasicrystalline structures: dynamical trace mapping, scaling and self-similarity of the spectrum. Journal of the Mechanics and Physics of Solids, 119:83-103, 2018.

[16] Lorenzo Morini, Zafar Gokay Tetik, Gal Shmuel, and Massimiliano Gei. On the universality of the frequency spectrum and band-gap optimization of quasicrystalline-generated structured rods. Philosophical Transactions of the Royal Society A, 378(2162):20190240, 2020.

[17] Mahmoud I. Hussein, Michael J. Leamy, and Massimo Ruzzene. Dynamics of Phononic Materials and Structures: Historical Origins, Recent Progress, and Future Outlook. Applied Mechanics Reviews, 66(4), 05 2014. 040802.

[18] Ben Lustig and Gal Shmuel. On the band gap universality of multiphase laminates and its applications. Journal of the Mechanics and Physics of Solids, 117:37-53, 2018.

[19] Witarto Witarto, Kalyana B Nakshatrala, and Yi-Lung Mo. Global sensitivity analysis of frequency band gaps in one-dimensional phononic crystals. Mechanics of Materials, 134:38$53,2019$.

[20] Xinnan Liu, Yiqiang Ren, Xiaoruan Song, and W Witarto. A global sensitivity analysis method based on the gauss-lobatto integration and its application in layered periodic foundations with initial stress. Composite Structures, 244:112297, 2020.

[21] Karl F Graff. Wave motion in elastic solids. Courier Corporation, 2012.

[22] Brian Hall. Lie groups, Lie algebras, and representations: an elementary introduction, volume 222. Springer, 2015.

[23] Marcel Novaes. Some basics of su $(1,1)$. Revista Brasileira de Ensino de Fisica, 26(4):351$357,2004$.

[24] Charles Kittel, Paul McEuen, and Paul McEuen. Introduction to solid state physics, volume 8. Wiley New York, 1996.

[25] Lawrence C. Evans. Partial differential equations. American Mathematical Society, Providence, R.I., 2010.

[26] James Kaklamanos, Laurie G Baise, Eric M Thompson, and Luis Dorfmann. Comparison of 1d linear, equivalent-linear, and nonlinear site response models at six kik-net validation sites. Soil Dynamics and Earthquake Engineering, 69:207-219, 2015.

[27] Yoshimitsu Okada, Keiji Kasahara, Sadaki Hori, Kazushige Obara, Shoji Sekiguchi, Hiroyuki Fujiwara, and Akira Yamamoto. Recent progress of seismic observation networks in japan-hinet, f-net, k-net and kik-net-. Earth, Planets and Space, 56(8):xv-xxviii, 2004.

[28] Stephen Wolfram. The mathematica book, volume 4. Cambridge University Press Cambridge, 2000 . 


\section{Appendix A. Derivation of EXaCt General expressions}

A.1. Review of multi-index notation. A binary multi-index $b \in(\{0,1\})^{N}$ is an $N$-tuple of numbers, in this case being either 0 or 1 . Its "degree" is equal to $|b|=b_{1}+\ldots+b_{N}$, thus, in this case, the degree is equal to the number of non-zero elements.

When a variable, call it $x \in \mathbb{R}^{N}$, is raised to the multi-index yields

$$
x^{\mathrm{b}}=x_{1}^{\mathrm{b}_{1}} \ldots x_{N}^{\mathrm{b}_{N}} .
$$

Note that a certain polynomial of degree $N, p(x) \in \mathbb{P}_{N}(\mathbb{R})$, can be written as

$$
p(x)=\sum_{\beta=0}^{\lfloor N / 2\rfloor} \sum_{|\mathrm{b}|=2 \beta} C_{\mathrm{b}} x^{\mathrm{b}} \text {. }
$$

So, for instance, if $N=3$ :

$$
\begin{aligned}
& \text { for }|\mathbf{b}|=0:\left\{x_{1}^{0} x_{2}^{0} x_{3}^{0}\right\}=\{1\}, \\
& \text { for }|\mathbf{b}|=2:\left\{x_{1}^{1} x_{2}^{1} x_{3}^{0}, x_{1}^{1} x_{2}^{0} x_{3}^{1}, x_{1}^{0} x_{2}^{1} x_{3}^{1}\right\}=\left\{x_{1} x_{2}, x_{1} x_{3}, x_{2} x_{3}\right\},
\end{aligned}
$$

hence, it defines the following polynomial:

$$
p(x)=\sum_{\beta=0}^{1} \sum_{|\mathrm{b}|=2 \beta} C_{\mathrm{b}} x^{\mathrm{b}}=C_{(0,0,0)}+C_{(1,1,0)} x_{1} x_{2}+C_{(1,0,1)} x_{1} x_{3}+C_{(0,1,1)} x_{2} x_{3} .
$$

Yet another example, if $N=4$ :

$$
\text { for }|\mathbf{b}|=0:\left\{x_{1}^{0} x_{2}^{0} x_{3}^{0} x_{4}^{0}\right\}=\{1\},
$$

$$
\begin{gathered}
\text { for }|\mathbf{b}|=2:\left\{x_{1}^{1} x_{2}^{1} x_{3}^{0} x_{4}^{0}, x_{1}^{1} x_{2}^{0} x_{3}^{1} x_{4}^{0}, x_{1}^{1} x_{2}^{0} x_{3}^{0} x_{4}^{1}, x_{1}^{0} x_{2}^{1} x_{3}^{1} x_{4}^{0}, x_{1}^{0} x_{2}^{1} x_{3}^{0} x_{4}^{1}, x_{1}^{0} x_{2}^{0} x_{3}^{1} x_{4}^{1}\right\} \\
= \\
=\left\{x_{1} x_{2}, x_{1} x_{3}, x_{1} x_{4}, x_{2} x_{3}, x_{2} x_{4}, x_{3} x_{4}\right\},
\end{gathered}
$$

$$
\text { for }|\mathbf{b}|=4:\left\{x_{1}^{1} x_{2}^{1} x_{3}^{1} x_{4}^{1}\right\}=\left\{x_{1} x_{2} x_{3} x_{4}\right\},
$$

and thus

$$
\begin{aligned}
p(x)= & \sum_{\beta=0}^{2} \sum_{|\mathbf{b}|=2 \beta} C_{\mathbf{b}} x^{\mathrm{b}}=C_{(0,0,0,0)}+C_{(1,1,0,0)} x_{1} x_{2}+C_{(1,0,1,0)} x_{1} x_{3}+C_{(1,0,0,1)} x_{1} x_{4} \\
(34) \quad+ & C_{(0,1,1,0)} x_{2} x_{3}+C_{(0,1,0,1)} x_{2} x_{4}+C_{(0,0,1,1)} x_{3} x_{4}+C_{(1,1,1,1)} x_{1} x_{2} x_{3} x_{4} .
\end{aligned}
$$

A.2. Dimensionless form of the matrix system. Again, consider a N-layer cell; assume different lengths $L_{i}, i=1, \ldots, N$. The total length of the cell is therefore $L=\sum_{i=1}^{N} L_{i}$.

Define a characteristic displacement $\mathcal{U}$, and a characteristic force $\sigma_{c h}=a_{c h} \cdot \mathcal{U} / L$, where the value of $a_{c h}$ can be thought to be the one of any of the layers. The longitudinal coordinate within the layer is parametrized using $\xi=x / L$. Using these new parameters, eq. (3) can be rewritten as

$$
\frac{d \tilde{\boldsymbol{f}}}{d \xi}=\frac{d}{d \xi}\left[\begin{array}{c}
\tilde{u} \\
\tilde{\sigma}
\end{array}\right]=\left[\begin{array}{cc}
0 & 1 / \gamma_{k} \\
-\eta_{k} r^{2} & 0
\end{array}\right]\left[\begin{array}{c}
\tilde{u} \\
\tilde{\sigma}
\end{array}\right]=\boldsymbol{A}_{k} \tilde{\boldsymbol{f}}(\xi)
$$


where $\tilde{u}=\hat{u} / \mathcal{U}, \tilde{\sigma}=\hat{\sigma} A / \sigma_{c h}$, and $\eta_{k}=\rho_{k} / \rho_{c h}, \zeta=\omega L / \sqrt{a_{c h} / \rho_{c h}}$.

At the bottom of the k-th layer, wherein $\xi-\xi_{\text {ref }}=\beta_{k}=L_{k} / L$,

$$
\begin{aligned}
\tilde{\boldsymbol{f}}\left(\xi_{k}\right) & =\exp \left(\left[\begin{array}{cc}
0 & \beta_{k} / \gamma_{k} \\
-\beta_{k} \eta_{k} r^{2} & 0
\end{array}\right]\right) \tilde{\boldsymbol{f}}\left(\xi_{k-1}\right) \\
& =\exp \left(\left[\begin{array}{cc}
0 & \alpha_{k} \\
\alpha_{k} R_{k}^{2} & 0
\end{array}\right]\right) \tilde{\boldsymbol{f}}\left(\xi_{k-1}\right) \\
& =\left[\begin{array}{cc}
\cosh \left(\alpha_{k} R_{k}\right) & R_{k}^{-1} \sinh \left(\alpha_{k} R_{k}\right) \\
R_{k} \sinh \left(\alpha_{k} R_{k}\right) & \cosh \left(\alpha_{k} R_{k}\right)
\end{array}\right] \tilde{\boldsymbol{f}}\left(\xi_{k-1}\right),
\end{aligned}
$$

where $\alpha_{k}=\beta_{k} / \gamma_{k}, \sinh \left(\alpha_{k} R_{k}\right)=\mathrm{i} \sin \left(\zeta_{k}\right)$ and $\cosh \left(\alpha_{k} R_{k}\right)=\cos \left(r_{k}\right)$, where $\zeta_{k}=$ $\omega h_{k} / \sqrt{\mu_{k} / \rho_{k}}$, and $R_{k}=\zeta \sqrt{-\gamma_{k} \eta_{k}}=\zeta \sqrt{-\rho_{k} \mu_{k} / \rho_{c h} a_{c h}}$.

A.3. Analogy with Lorentz transformations. Compare the last expression to the Lorentz transformation realized with a $2 \times 2$ matrix of a "boost" of magnitude $\tau_{0}$ along the direction $\phi_{0}, \mathrm{~T}_{\tau_{0}, \phi_{0}}[23]$,

$$
\mathrm{T}_{\tau_{0}, \phi_{0}}=\left[\begin{array}{cc}
\cosh \left(\tau_{0} / 2\right) & e^{-\mathrm{i} \phi_{0}} \sinh \left(\tau_{0} / 2\right) \\
e^{\mathrm{i} \phi_{0}} \sinh \left(\tau_{0} / 2\right) & \cosh \left(\tau_{0} / 2\right)
\end{array}\right] .
$$

We will use an analogy to this type of transformations as stepping stone to understand the action of the transfer matrices.

The superposition of any number of layers can be seen as a composition of any number of successive Lorentz boosts. It is a well-known fact that the composition of boosts is not a boost, but a composition of a rotation and a boost [23]; this can be regarded as a re-statement of an obvious result as to homogeneization : a set of continuum layers cannot be homogeneized into a single equivalent layer for the purpose of arbitrary-wavelength wave propagation (such homogeneization is only feasible in the limit of small wavelengths, see the reference for more details).

In any case, the boost can be represented by matrices of the type

$$
\left[\begin{array}{ll}
\Upsilon_{1} & \bar{\Upsilon}_{2} \\
\Upsilon_{2} & \bar{\Upsilon}_{1}
\end{array}\right]
$$

where - amounts to the conjugation. If we move to projective coordinates (hence we can re-scale the entries of the matrix freely as long as all of them are scaled by the same factor) the action over a vector can be thus realized by means of fractional transformations, i.e., given an starting element $z$, the new element, according to the transformation defined by eq. (38), $z^{\prime}$ is

$$
z^{\prime}=\frac{\Upsilon_{1} z+\Upsilon_{2}}{\bar{\Upsilon}_{2} z+\bar{\Upsilon}_{1}}
$$

which, in the matrix form is expressed as

$$
[z, 1]^{\top}\left[\begin{array}{ll}
\Upsilon_{1} & \bar{\Upsilon}_{2} \\
\Upsilon_{2} & \bar{\Upsilon}_{1}
\end{array}\right]
$$

the term 1 actually gives away that we pass to work over the projective line. See that if all the entries of the boost matrix are multiplied by the same factor, the transformation does not change eq. (39). The expression of the boost in projective coordinates is therefore

$$
\mathrm{T}_{\tau_{0}, \phi_{0}}(\Upsilon)=\frac{\left(\cosh \tau_{0} / 2\right) \zeta+e^{\mathrm{i} \phi_{0}} \sinh \tau_{0} / 2}{\left(e^{-\mathrm{i} \phi_{0}} \sinh \tau_{0} / 2\right) \Upsilon+\cosh \tau_{0} / 2} .
$$


See how, in general, these transformations are not commutative (the order in which successive transformations are effectuated is critical to the final result).

Pushing the analogy, we claim that eq. (36), up to scaling by a constant, can be expressed as

$$
\tilde{\boldsymbol{f}}\left(\xi_{k}\right)=\frac{\cosh \left(\alpha_{k} R_{k}\right) \tilde{\boldsymbol{f}}\left(\xi_{k-1}\right)+R_{k} \sinh \left(\alpha_{k} R_{k}\right)}{R_{k}^{-1} \sinh \left(\alpha_{k} R_{k}\right) \tilde{\boldsymbol{f}}\left(\xi_{k-1}\right)+\cosh \left(\alpha_{k} R_{k}\right)}=\frac{\tilde{\boldsymbol{f}}\left(\xi_{k-1}\right)+R_{k} \tanh \left(\alpha_{k} R_{k}\right)}{R_{k}^{-1} \tanh \left(\alpha_{k} R_{k}\right) \tilde{\boldsymbol{f}}\left(\xi_{k-1}\right)+1},
$$

wherein, abusing the notation, now $\tilde{\boldsymbol{f}}$ is understood as a complex number. As we wish to find a recursion formula when combining $N$ successive boosts, let us put the previous formula in a more compact form:

$$
\tilde{\boldsymbol{f}}\left(\xi_{k}\right)=\frac{c_{k} \tilde{\boldsymbol{f}}\left(\xi_{k-1}\right)+s_{k}}{s_{k}^{*} \tilde{\boldsymbol{f}}\left(\xi_{k-1}\right)+c_{k}^{*}}
$$

wherein the asterisk indicates that the tanh term is divided by the corresponding $R_{k}$. The asterisk is also added to the other term to facilitate appraising the recursion, it is understood to change nothing in that case.

\section{A.4. Obtaining the general recursion of the cumulative transfer matrix.}

A.4.1. Particular case: one-layer system. In this case, simply use eq. (43):

$$
\begin{aligned}
\boldsymbol{f}(1) & =\frac{c_{1} \boldsymbol{f}(0)+s_{1}}{s_{1}^{*} \boldsymbol{f}(0)+c_{1}^{*}} \\
& =\frac{\cos \left(\zeta_{1}\right) \boldsymbol{f}(0)+\sqrt{-\rho_{1} a_{1} / \rho_{c h} a_{c h}} \mathrm{i} \sin \left(\zeta_{1}\right)}{\frac{\mathrm{i} \sin \left(\zeta_{1}\right)}{\sqrt{-\rho_{1} a_{1} / \rho_{c h} a_{c h}}} \boldsymbol{f}(0)+\cos \left(\zeta_{1}\right)} \\
& =\frac{\boldsymbol{f}(0)-\sqrt{\frac{\rho_{1} a_{1}}{\rho_{c h} a_{c h}}} \tan \left(\zeta_{1}\right)}{\sqrt{\frac{\rho_{c h} a_{c h}}{\rho_{1} a_{1}}} \tan \left(\zeta_{1}\right) \boldsymbol{f}(0)+1},
\end{aligned}
$$

thus we can state also

$$
\begin{aligned}
& c_{1}=c_{1}^{*}=1, \\
& s_{1}=-\sqrt{\frac{\rho_{1} a_{1}}{\rho_{c h} a_{c h}}} \tan \left(\zeta_{1}\right), \\
& s_{1}^{*}=\sqrt{\frac{\rho_{c h} a_{c h}}{\rho_{1} a_{1}}} \tan \left(\zeta_{1}\right)
\end{aligned}
$$

Hence, after scaling back by the cosine, in the simplest case we obtain

$$
\frac{1}{2} \operatorname{trace}(\boldsymbol{T})=\frac{1}{2} \cos \left(\frac{\omega L_{1}}{a_{1}}\right)\left(c_{1}+c_{1}^{*}\right)=\cos \left(\frac{\omega L_{1}}{a_{1}}\right),
$$

which means that the dispersion relation in a homogeneous layer presents no bandgaps. 
A.4.2. Particular case: two-layer system. Perform two consecutive fractional transformations in this case,

$$
\begin{aligned}
\boldsymbol{f}\left(\beta_{1}+\beta_{2}\right) & =\boldsymbol{f}(1)=\frac{c_{2} \boldsymbol{f}\left(\beta_{1}\right)+s_{2}}{s_{2}^{*} \boldsymbol{f}\left(\beta_{1}\right)+c_{2}^{*}}=\frac{c_{2} \frac{c_{1} \boldsymbol{f}(0)+s_{1}}{s_{1}^{*} \boldsymbol{f}(0)+c_{1}^{*}}+s_{2}}{s_{2}^{*} \frac{c_{1} \boldsymbol{f}(0)+s_{1}}{s_{1}^{*} \boldsymbol{f}(0)+c_{1}^{*}}+c_{2}^{*}} \\
& =\frac{\left(c_{2} c_{1}+s_{2} s_{1}^{*}\right) \boldsymbol{f}(0)+\left(s_{2} c_{1}^{*}+c_{2} s_{1}\right)}{\left(s_{2}^{*} c_{1}+c_{2}^{*} s_{1}^{*}\right) \boldsymbol{f}(0)+\left(c_{2}^{*} c_{1}^{*}+s_{2}^{*} s_{1}\right)},
\end{aligned}
$$

then, scaling back multiplying by two cosines,

$$
\frac{1}{2} \operatorname{trace}(\boldsymbol{T})=\frac{1}{2} \cos \left(\frac{\omega L_{1}}{a_{1}}\right) \cos \left(\frac{\omega L_{2}}{a_{2}}\right)\left[\left(c_{2} c_{1}+s_{2} s_{1}^{*}\right)+\left(c_{2}^{*} c_{1}^{*}+s_{2}^{*} s_{1}\right)\right],
$$

For the purpose of easing notation as we push the recurrence forward, let us call

$$
\begin{aligned}
& c_{2,1}=c_{2} c_{1}+s_{2} s_{1}^{*}=1-\sqrt{\frac{\rho_{1} a_{1}}{\rho_{2} a_{2}}} \tan \left(\zeta_{1}\right) \tan \left(\zeta_{2}\right), \\
& c_{2,1}^{*}=c_{2}^{*} c_{1}^{*}+s_{2}^{*} s_{1}=1-\sqrt{\frac{\rho_{2} a_{2}}{\rho_{1} a_{1}}} \tan \left(\zeta_{1}\right) \tan \left(\zeta_{2}\right), \\
& s_{2,1}=s_{2} c_{1}^{*}+c_{2} s_{1}=-\sqrt{\frac{\rho_{2} \mu_{2}}{\rho_{c h} a_{c h}}} \tan \left(\zeta_{2}\right)-\sqrt{\frac{\rho_{1} a_{1}}{\rho_{c h} a_{c h}}} \tan \left(\zeta_{1}\right), \\
& s_{2,1}^{*}=s_{2}^{*} c_{1}+c_{2}^{*} s_{1}^{*}=\sqrt{\frac{\rho_{c h} a_{c h}}{\rho_{2} a_{2}}} \tan \left(\zeta_{2}\right)+\sqrt{\frac{\rho_{c h} a_{c h}}{\rho_{1} a_{1}}} \tan \left(\zeta_{1}\right),
\end{aligned}
$$

see how the "c-terms" accumulate two tangent factors in one addend at once while the "s-terms" contain two terms with one tangent each. This structure persists as more layers are stacked.

A.4.3. $N>2$ number of layers. For the general case, just set a recurrence up from eq. (49):

$$
\begin{aligned}
& c_{i, 1}=c_{i} c_{i-1,1}+s_{i} s_{i-1,1}^{*}, \\
& c_{i, 1}^{*}=c_{i}^{*} c_{i-1,1}^{*}+s_{i}^{*} s_{i-1,1}, \\
& s_{i, 1}=s_{i} c_{i-1,1}^{*}+c_{i}^{*} s_{i-1,1}, \\
& s_{i, 1}^{*}=s_{i}^{*} c_{i-1,1}+c_{i} s_{i-1,1}^{*},
\end{aligned}
$$

and proceed to "unzip" it to see that (the scaling-back by the cosines already included)

$$
T_{11}=c_{1, N}=\left(\prod_{i=1}^{N} \cos \left(r_{i}\right)\right)\left(\sum_{\beta=0}^{\lfloor N / 2\rfloor} \sum_{|\mathrm{b}|=2 \beta} K_{\mathrm{b}} \tan (r)^{\mathrm{b}}\right),
$$

while the coefficients

$$
K_{\mathrm{b}}=(-1)^{|\mathbf{b}| / 2}(\sqrt{\rho \mu})^{\mathrm{f}(\mathbf{b})},
$$

where $\mathrm{b} \in(0,1)^{N}$ and has been presented in the body of the text (and it is reintroduced later below). 
The coefficients with asterisk are given by the same expression but inverting the rations forming the coefficients, thus

$$
T_{22}=s_{1, N}=\left(\prod_{i=1}^{N} \cos \left(r_{i}\right)\right)\left(\sum_{\beta=0}^{\lfloor N / 2\rfloor} \sum_{|\mathbf{b}|=2 \beta} L_{\mathbf{b}} \tan (r)^{\mathbf{b}}\right),
$$

while the coefficients in this case

$$
L_{\mathrm{b}}=(-1)^{|\mathbf{b}| / 2}(\sqrt{\rho \mu})^{-\mathrm{f}(\mathbf{b})} .
$$

The general recursion yields the following expression for the half-trace:

$$
\frac{1}{2} \operatorname{trace}(\boldsymbol{T}(L, 0))=\frac{T_{11}+T_{22}}{2}=\left(\prod_{i=1}^{N} \cos \left(r_{i}\right)\right)\left(1-\sum_{\beta=1}^{\lfloor N / 2\rfloor} \sum_{|\mathrm{b}|=2 \beta} \gamma_{\mathrm{b}} \tan (r)^{\mathrm{b}}\right),
$$

each $\mathrm{b} \in(\{0,1\})^{N}$ is a multi-index representing one permutation out of $\left(\begin{array}{c}N \\ 2 \beta\end{array}\right)$ possible ones having $|\mathrm{b}|=2 \beta$ terms equal to one while the other ones are zero, the coefficients $C_{\mathrm{b}}$ being given by

$$
\gamma_{\mathbf{b}}=\frac{(-1)^{|\mathbf{b}| / 2}}{2}\left[(\sqrt{\rho a})^{\mathrm{f}(\mathbf{b})}+(\sqrt{\rho a})^{-\mathrm{f}(\mathbf{b})}\right] .
$$

where the map $\mathrm{f}:(\{0,1\})^{N} \rightarrow(\{-1,0,1\})^{N}$ takes the multi-index b, entry-wise, to the multi-index $\mathrm{f}(\mathrm{b})$ defined as:

- if $\mathrm{b}_{i}=0$, then $\mathrm{f}\left(\mathrm{b}_{i}\right)=0$,

- if $\mathrm{b}_{i}=1$ and the previous value assigned by $\mathrm{f}$ to the prior 1-entry in $\mathrm{b}$ was -1 , then $\mathrm{f}\left(\mathrm{b}_{i}\right)=1$, else $\mathrm{f}\left(\mathrm{b}_{i}\right)=-1$.

Through this auxiliary multi-index $\mathrm{f}(\mathrm{b})$ the impedance contrasts enter the results.

The design example in Section 4 outlines how the multi-indices are constructed and how in turn they define the half-trace function. The first addends, those that go with $\mathrm{f}(\mathrm{b})$, correspond to $T_{11}$ while $T_{22}$ provides those with $-\mathrm{f}(\mathrm{b})$ see Appendix.

Resorting to the product-to-sum identity, the right-hand side of Equation (55) can be converted into a sum of $2^{N}$ products, each one of them being a product of $N$ functions, either sines or cosines. The identity states:

$$
\prod_{i=1}^{N} \cos \left(\theta_{i}\right)=\frac{1}{2^{N}} \sum_{\mathbf{e}^{\prime} \in(\{-1,1\})^{N}} \cos \left(\sum_{j=1}^{N} \mathbf{e}_{j}^{\prime} \theta_{j}\right),
$$

for any arguments $\theta_{i}, i=1, \ldots, N$.

Since all the sines that appear can be considered as cosines with extra phase $-\pi / 2$, they would also fit in the identity. Even more remarkable is the fact that, given that the degree of the multi-index bis always even, these sine terms (regarded as out-of-phase cosines) entail either a change of sign or nothing at all, so the final result will be a sum of cosine functions without phase-delay terms.

Summarizing, the half-trace function in Equation (55) admits the following form

$$
\frac{1}{2} \operatorname{trace}(\boldsymbol{T}(L, 0))=\sum_{k=1}^{2^{N}} \mathcal{T}_{k} \cos \left(\tau_{k} \omega\right)
$$

Finally, if we take advantage of the the fact that the cosine does not change if we swap the sign of the argument, we can half the number of harmonics as each 
harmonic appears effectively twice in eq. (58). Thus we introduce the multi-index e whose first entry is always equal to one, $e_{1}=1$. 

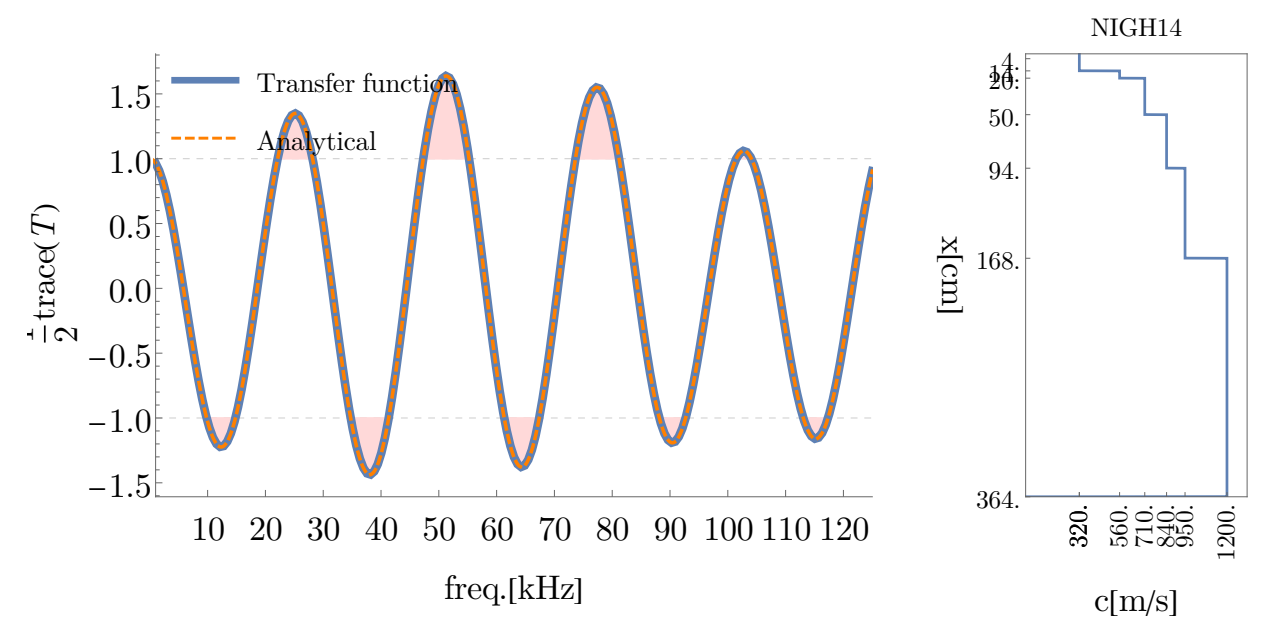

Figure 13. Dispersion diagram (left panel) and spectrum of halftrace (right panel) of rod having properties (right panel) that mirror Kik-Net site NIGH14 (shaded region in left panel corresponds to bandgap).

Appendix B. Extra figures for other rod profiles
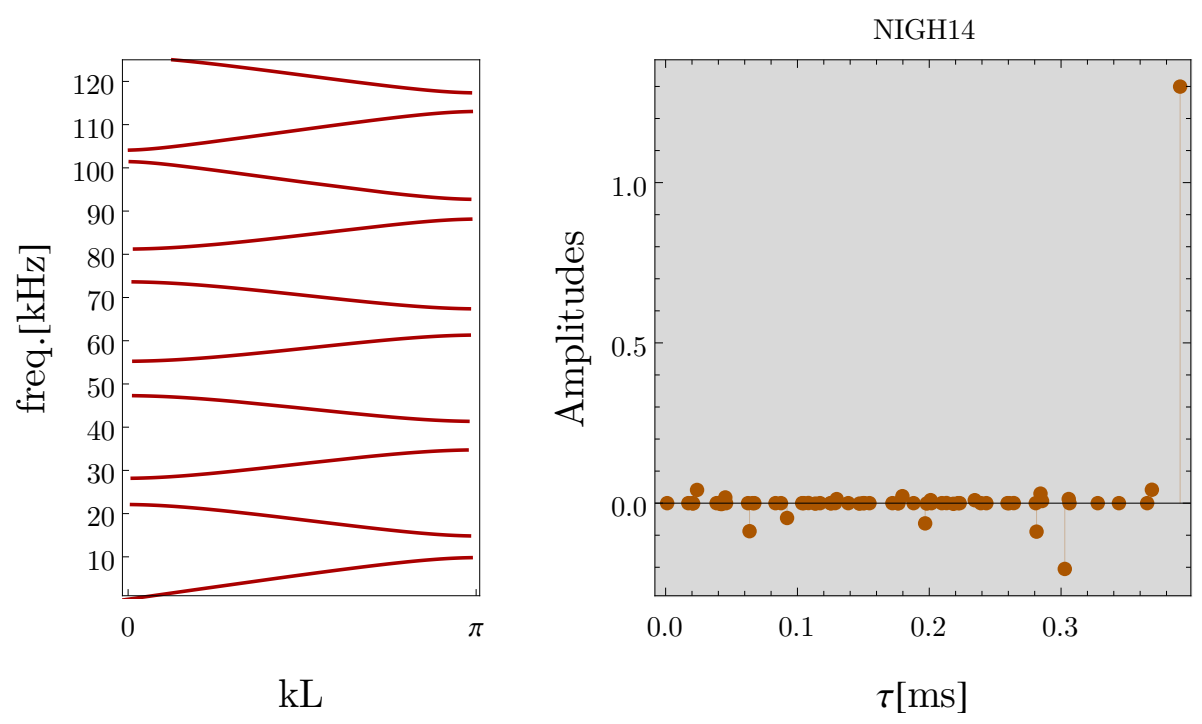

FiguRe 14. Dispersion diagram (left panel) and spectrum of halftrace (right panel) of rod having properties (right panel) that mirror Kik-Net site NIGH14. 

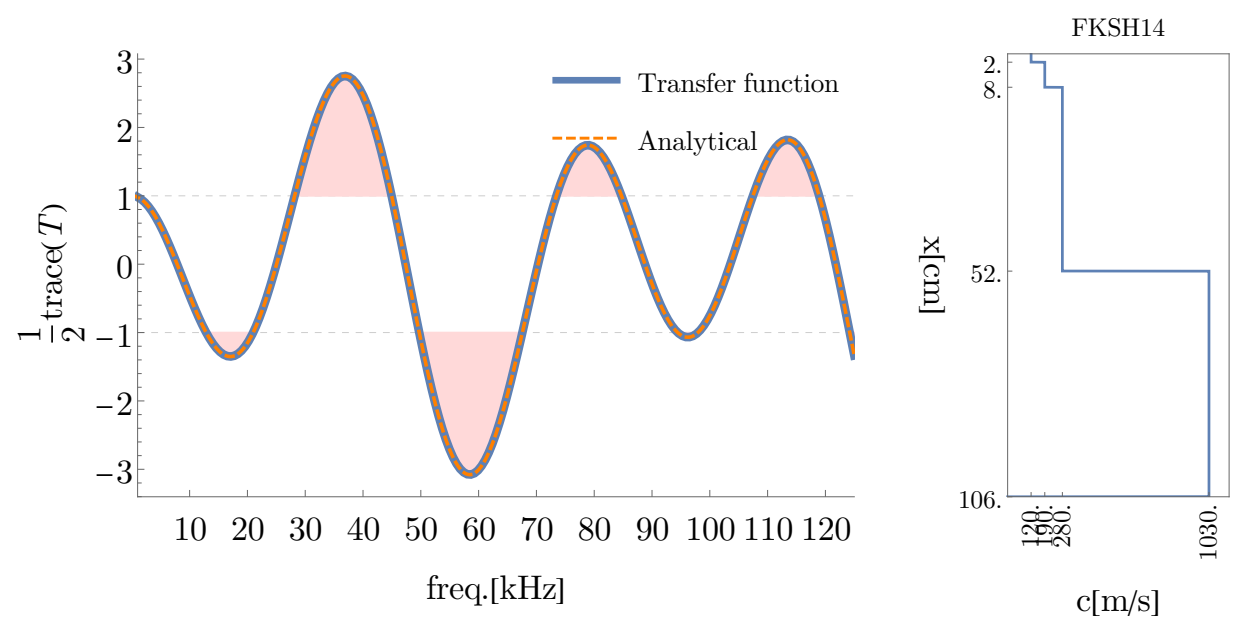

Figure 15. Dispersion diagram (left panel) and spectrum of halftrace (right panel) of rod having properties (right panel) that mirror Kik-Net site FHSK14 (shaded region in left panel corresponds to bandgap).
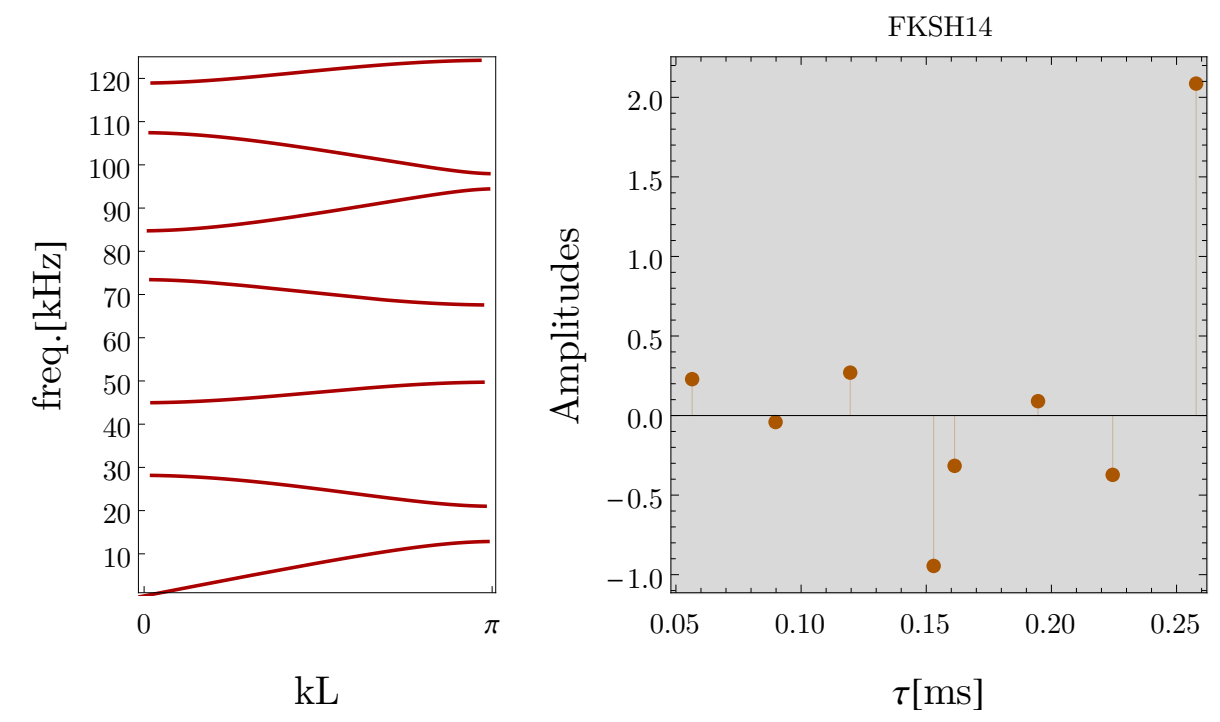

Figure 16. Dispersion diagram (left panel) and spectrum of halftrace (right panel) of rod having properties (right panel) that mirror Kik-Net site FHSK14. 

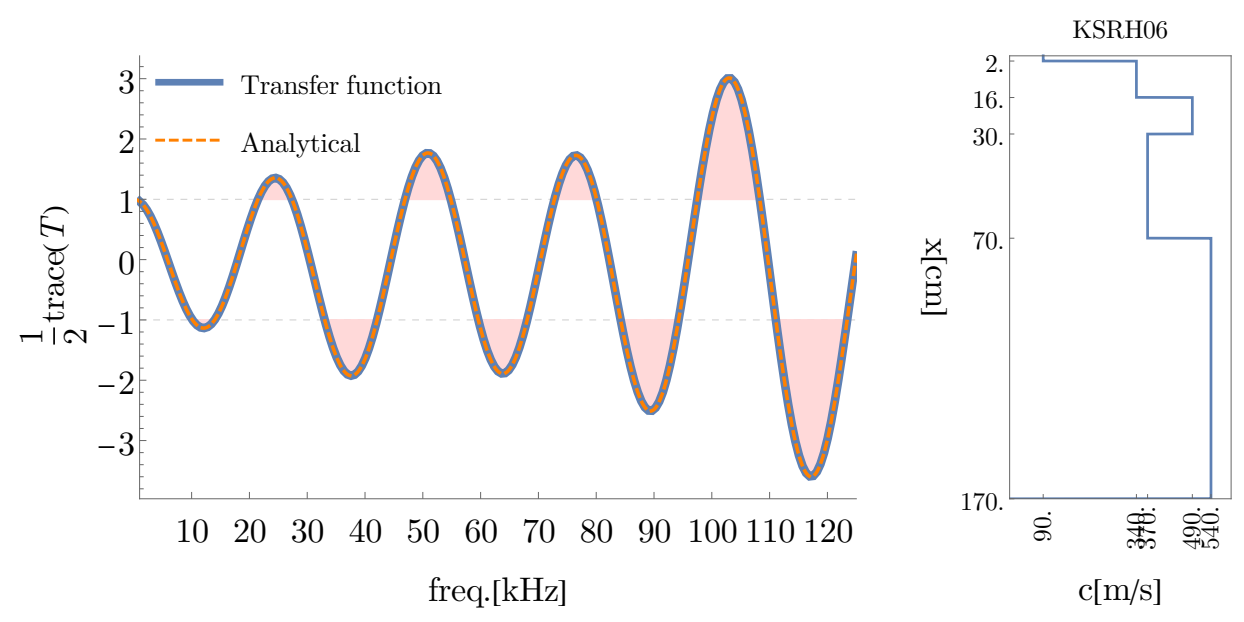

Figure 17. Dispersion diagram (left panel) and spectrum of halftrace (right panel) of rod having properties (right panel) that mirror Kik-Net site KSRH06 (shaded region in left panel corresponds to bandgap).

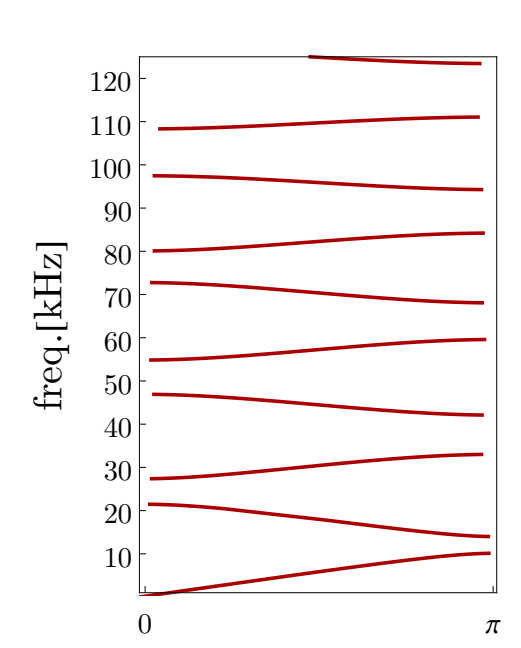

$\mathrm{kL}$

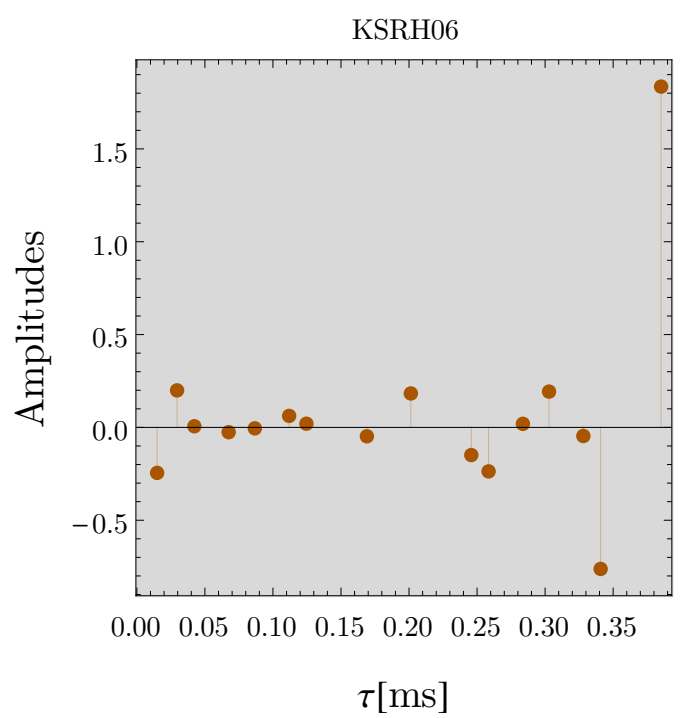

Figure 18. Dispersion diagram (left panel) and spectrum of halftrace (right panel) of rod having properties (right panel) that mirror Kik-Net site KSRH06. 

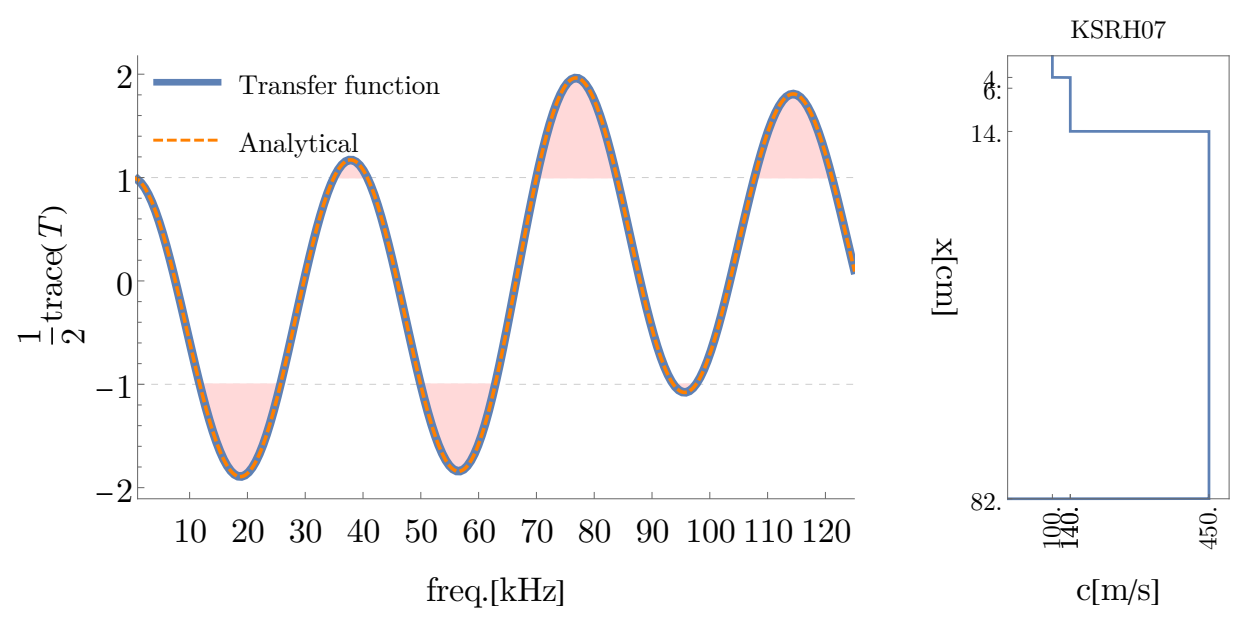

Figure 19. Dispersion diagram (left panel) and spectrum of halftrace (right panel) of rod having properties (right panel) that mirror Kik-Net site KSRH07 (shaded region in left panel corresponds to bandgap).
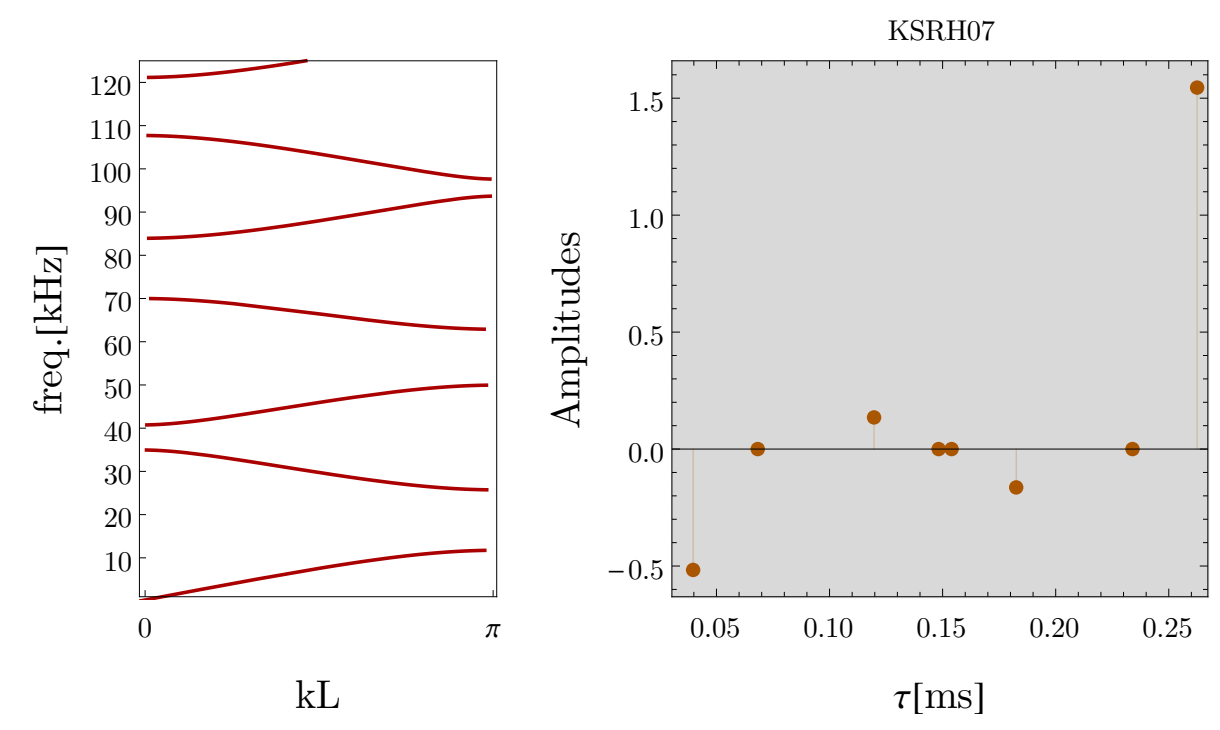

Figure 20. Dispersion diagram (left panel) and spectrum of halftrace (right panel) of rod having properties (right panel) that mirror Kik-Net site KSRH07. 

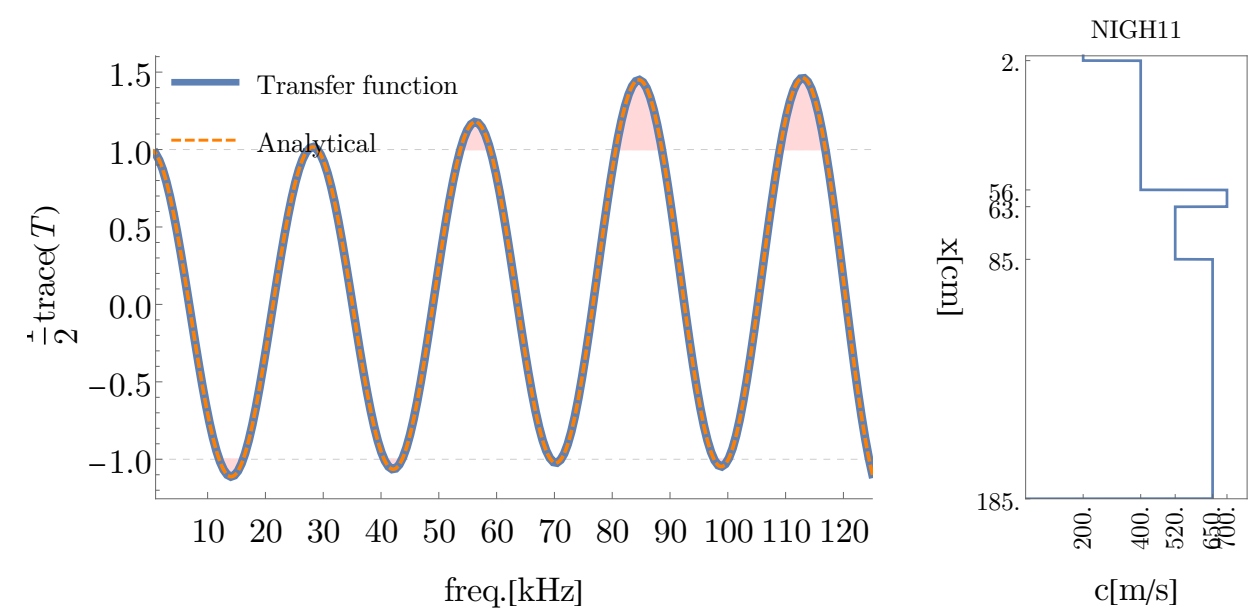

Figure 21. Dispersion diagram (left panel) and spectrum of halftrace (right panel) of rod having properties (right panel) that mirror Kik-Net site NIGH11 (shaded region in left panel corresponds to bandgap).

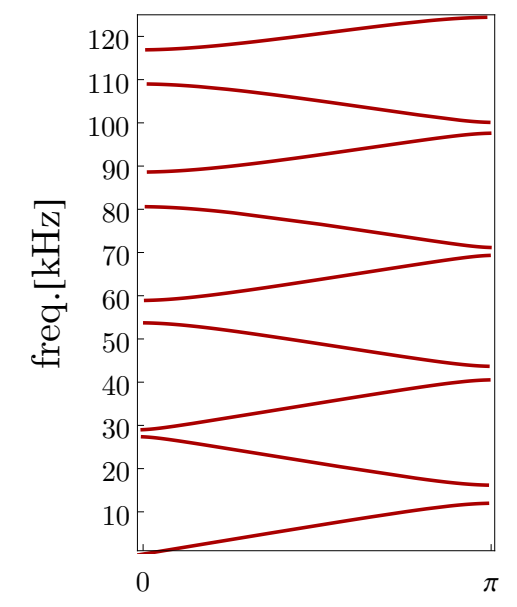

$\mathrm{kL}$

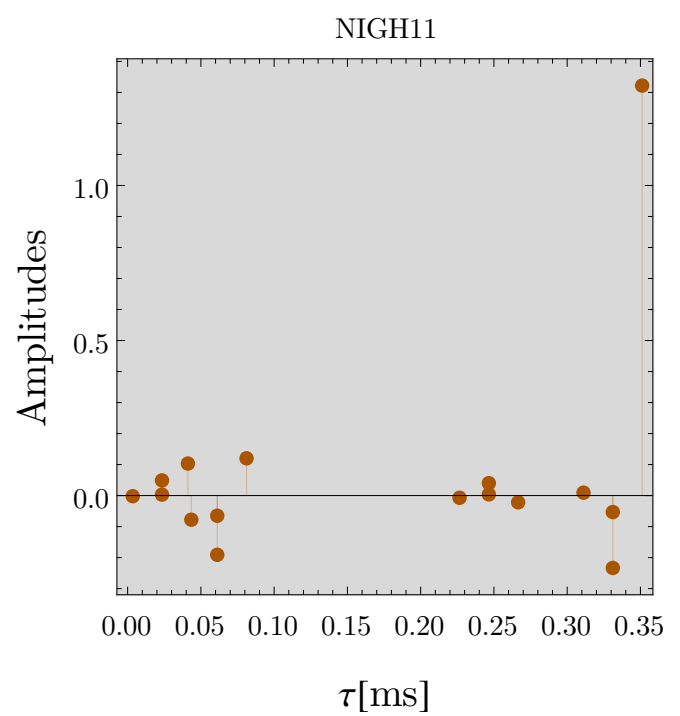

Figure 22. Dispersion diagram (left panel) and spectrum of halftrace (right panel) of rod having properties (right panel) that mirror Kik-Net site NIGH11. 

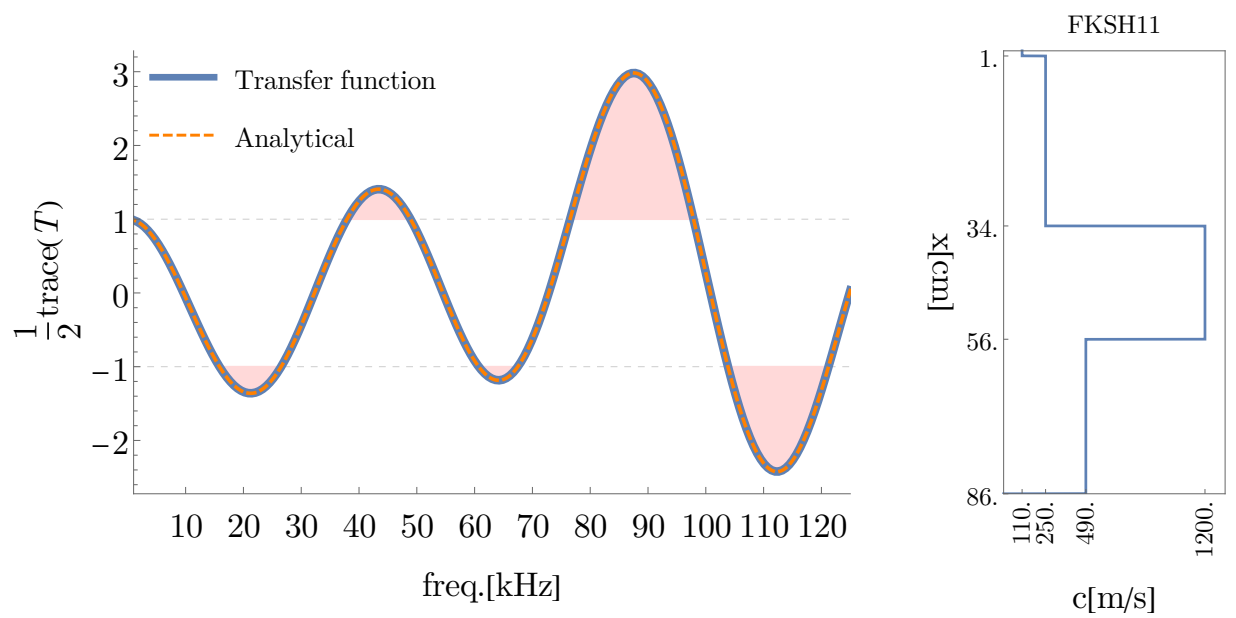

Figure 23. Dispersion diagram (left panel) and spectrum of halftrace (right panel) of rod having properties (right panel) that mirror Kik-Net site FKSH11 (shaded region in left panel corresponds to bandgap).
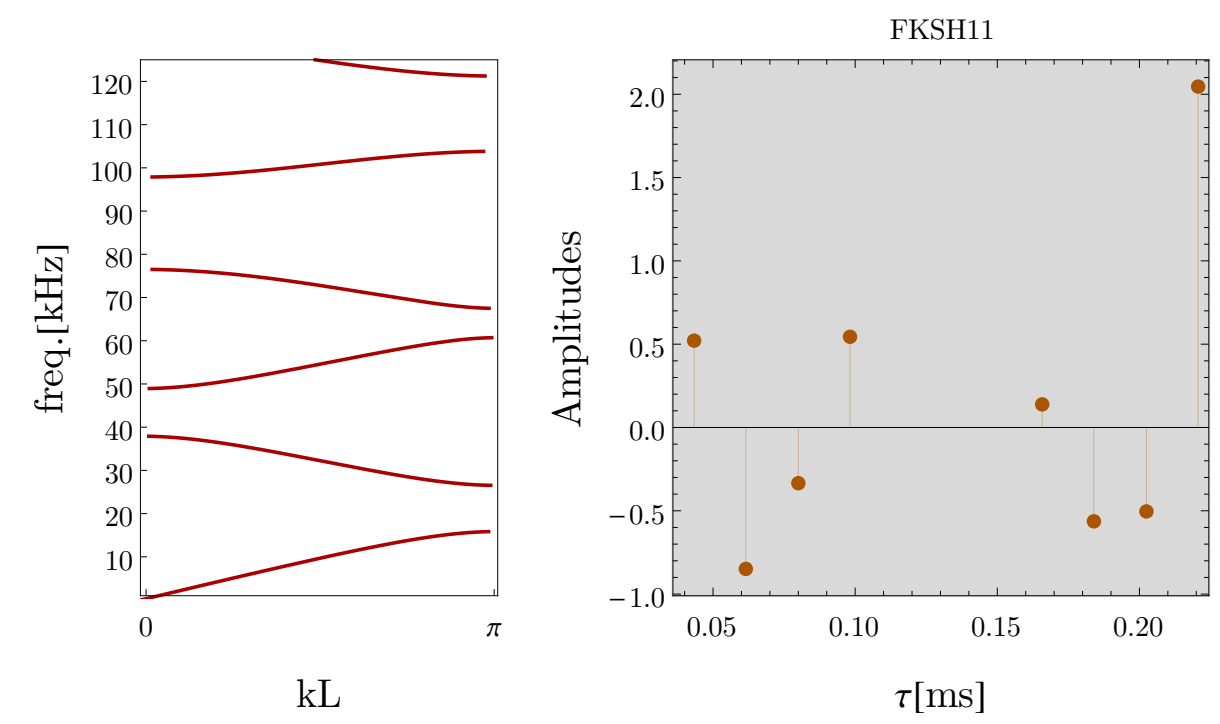

Figure 24. Dispersion diagram (left panel) and spectrum of halftrace (right panel) of rod having properties (right panel) that mirror Kik-Net site FKSH11. 

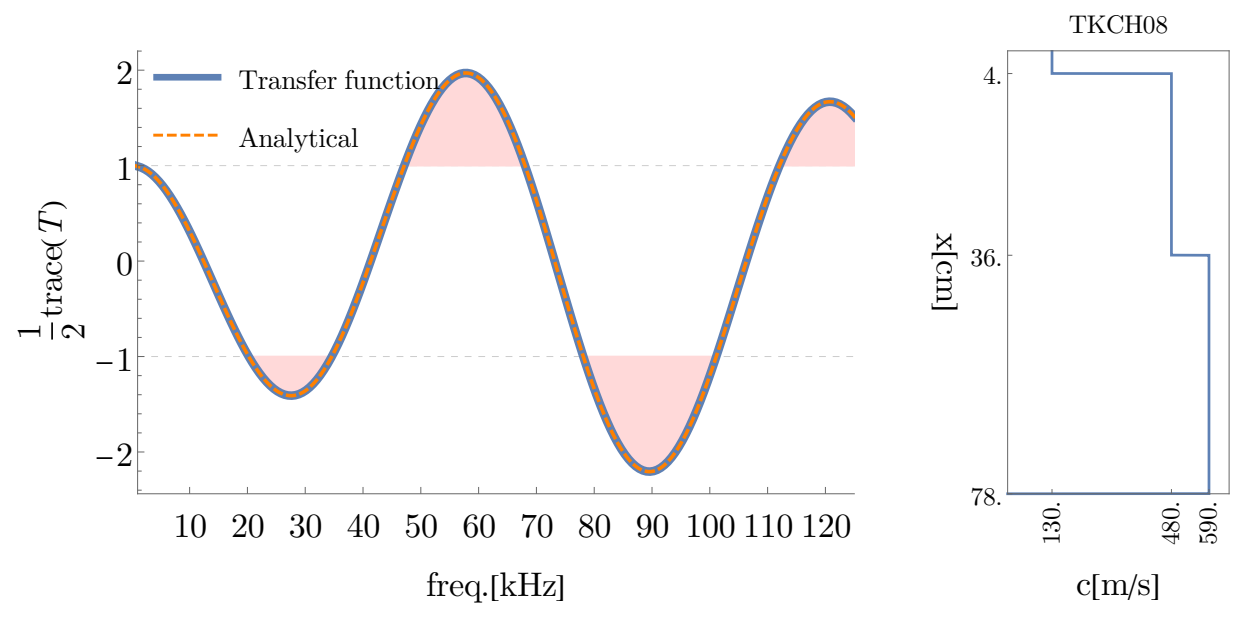

Figure 25. Dispersion diagram (left panel) and spectrum of halftrace (right panel) of rod having properties (right panel) that mirror Kik-Net site TKCH08 (shaded region in left panel corresponds to bandgap).
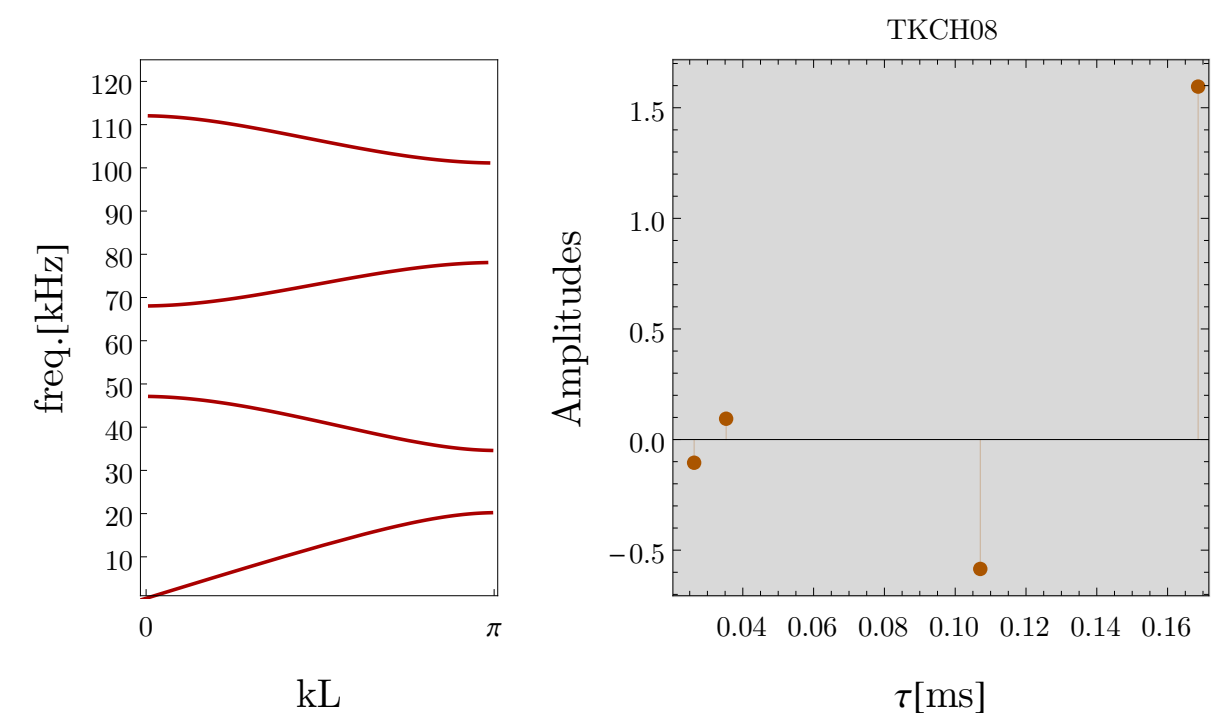

Figure 26. Dispersion diagram (left panel) and spectrum of halftrace (right panel) of rod having properties (right panel) that mirror Kik-Net site TKCH08. 
Civil Engineering Institute École Politechnique Fédérale de Lausanne (EPFL), LauSANNe, SwitzerLand

Email address: joaquin.garciasuarez@epfl.ch 